\title{
ON THE STRUCTURE OF PEDERSEN-POON TWISTOR SPACES
}

\author{
NOBUHIRO HONDA
}

\begin{abstract}
We study algebro-geometric properties of certain twistor spaces over $n \mathrm{CP}^{2}$ with two dimensional torus actions, whose existence was proved by Pedersen and Poon [16]. We show that they have a pencil whose general members are non-singular toric surface, and completely determine the structure of the reducible members of the pencil, which are also toric surfaces. In the course of our proof, we describe behaviors of the above pencil under equivariant smoothing. Relation between the weighted dual graphs of the toric surfaces in the pencil and similar invariant of the above torus action on $n \mathrm{CP}^{2}$ is also determined.
\end{abstract}

\section{Introduction}

An oriented four-dimensional Riemannian manifold $(M, g)$ is said to be selfdual iff the anti-self-dual part of the Weyl curvature of $g$ is identically zero. The four sphere with the standard metric and the complex projective plane with the Fubini-Study metric are basic examples of compact self-dual manifolds.

Until the late of 1980's, only a few examples were known of compact selfdual manifolds. In 1989 Donaldson and Friedman [3] gave a general method for constructing self-dual metrics on the connected sum of compact self-dual manifolds. Their method relies on the twistor theory and deformation theory of compact complex spaces with only simple normal crossing singularities: let $\left(M_{1}, g_{1}\right)$ and $\left(M_{2}, g_{2}\right)$ be compact self-dual manifolds and, $Z_{1}$ and $Z_{2}$ their associated twistor spaces respectively, which are three-dimensional compact complex manifolds foliated by non-singular rational curves parameterized by the four-manifolds $M_{i}$. Let $\Theta_{Z_{i}}(i=1,2)$ be the tangent sheaf of $Z_{i}$. Then one of their result ([3, Theorem 6.1]) is: if $H^{2}\left(Z_{1}, \Theta_{Z_{1}}\right)=H^{2}\left(Z_{2}, \Theta_{Z_{2}}\right)=0$, then certain singular compact complex space $Z^{\prime}$ which is constructed from $Z_{1}$ and $Z_{2}$ (and with only simple normal crossing singularities), can be deformed into a non-singular threefold which is a twistor space over $M_{1} \# M_{2}$. That is, there exists a self-dual metric on $M_{1} \# M_{2}$ (= the connected sum of $M_{1}$ and $M_{2}$ ). As a corollary, they proved that for any positive integer $n$ there exist self-

Received October 8, 1999. 
dual metrics on $n \mathrm{CP}^{2}$, where $n \mathrm{CP}^{2}$ denotes the connected sum of $n$ complex projective planes.

Later, Pedersen and Poon [16] developed an equivariant version of the above result of Donaldson and Friedman: let $\left(M_{1}, g_{1}\right)$ and $\left(M_{2}, g_{2}\right)$ be compact selfdual manifolds as above, and $G$ a compact Lie group acting on both of $\left(M_{1}, g_{1}\right)$ and $\left(M_{2}, g_{2}\right)$ isometrically. Assume that there exist $G$-fixed points $p_{i} \in M_{i}$ $(i=1,2)$ and an orientation reversing linear isometry between $T_{p_{1}} M_{1}$ and $T_{p_{2}} M_{2}$ which is $G$-equivariant with respect to the isotropic representations at $p_{i}$. Then $M_{1} \# M_{2}$ has a natural $G$-action. Under these circumstances, Pedersen and Poon [16, Theorem 4.4] proved that if $H^{2}\left(Z_{1}, \Theta_{Z_{1}}\right)=H^{2}\left(Z_{2}, \Theta_{Z_{2}}\right)=0$, then $M_{1} \# M_{2}$ has a self-dual metric with an isometric $G$-action.

Applying this result inductively for $\mathrm{CP}^{2}$, $\mathrm{s}$ with the standard $T^{2}:=S^{1} \times S^{1}$ action, they proved (cf. [16, Theorem 7.1]), with the aid of a result of Orlik and Raymond [14], that for any $n \geq 0$ and any effective $T^{2}$-action $\alpha: T^{2} \times n \mathrm{CP}^{2} \rightarrow$ $n \mathrm{CP}^{2}$, there exists a self-dual metric (i.e. a twistor space) on $n \mathrm{CP}^{2}$ with respect to which $\alpha$ is an isometric action. We call the corresponding twistor space over $n \mathrm{CP}^{2}$ Pedersen-Poon twistor spaces associated to $\alpha$ (cf. $\S 1.3$ for the precise definition). We note that effective $T^{2}$-actions on $n \mathrm{CP}^{2}$ are classified by certain combinatorial data [14], [10]. By construction, Pedersen-Poon twistor spaces $Z$ associated to $\alpha$ has a holomorphic $T^{2}$-action such that the twistor fibration $\pi: Z \rightarrow n \mathrm{CP}^{2}$ becomes a $T^{2}$-equivariant map. The purpose of the present paper is to study algebro-geometric properties of these twistor spaces in detail.

To state our result, we recall the following facts: any twistor space $Z$ associated to a compact simply connected self-dual four-manifold $(M, g)$ has a unique holomorphic line bundle $F$ which is characterized by the property that $F^{\otimes 2} \simeq-K_{Z}$ (=the anti-canonical bundle of $Z$ ). When a Lie group $G$ acts on $(M, g)$ isometrically, then $G$ acts holomorphically not only on $Z$ but also on the line bundle $F$. Let $H^{0}(Z, F)^{G}$ denote the linear subspace of $H^{0}(Z, F)$ generated by all $G$-fixed sections, and $|F|^{G} \subseteq|F|$ the linear subsystem whose corresponding linear subspace is $H^{0}(Z, F)^{G}$. Then our main result is the following:

MAIN TheOREM. Let $\alpha: T^{2} \times n \mathrm{CP}^{2} \rightarrow n \mathrm{CP}^{2}$ be any effective $T^{2}$-action and $Z$ any Pedersen-Poon twistor space associated to $\alpha$. Then all of (i)-(iv) below hold.

(i) $|F|^{T^{2}}$ is one-dimensional as a linear system, and general members of $|F|^{T^{2}}$ are biholomorphic to an irreducible non-singular toric surface $S$.

(ii) The number of reducible members of $|F|^{T^{2}}$ is $n+2$, each of which are of the form $D_{i}+\sigma\left(D_{i}\right)(1 \leq i \leq n+2)$, where every $D_{i}(1 \leq i \leq n+2)$ is irreducible non-singular toric surface and $\sigma$ is the real structure of $Z$.

(iii) The base locus of the pencil $|F|^{T^{2}}$ coincides with the (unique) torus 
invariant anticanonical curve of $S$ in (i).

(iv) The toric surfaces $S$ and $D_{i}(1 \leq i \leq n+2)$ in (i) and (ii) are uniquely determined by the original action $\alpha$ on $n \mathrm{CP}^{2}$.

Recall that non-singular toric surfaces are completely described by their weighted dual graphs [13, p. 44]. On the other hand, as shown in [14], effective $T^{2}$-actions on $n \mathrm{CP}^{2}$ are also classified by similar (weighted dual) graphs. We also determine the relationship between the weighted dual graph of $S$ (and $D_{i}$ ) in the above theorem and that of $\alpha$.

This paper consists of five sections. Section 1 summaries known results about the classification of $T^{2}$-actions on $n \mathrm{CP}^{2}(\$ 2.1)$ and the theory of toric surface (\$2.2), and gives a precise definition of Pedersen-Poon twistor spaces (§2.3). Also given are some lemmas needed in the subsequent sections. Section 2 proves that (ii) and (iii) of Main Theorem follow from (i). Section 3 provides the first step to prove (i) of Main Theorem. Certain singular space $Z^{\prime}$ and two families $\mathscr{P}_{1}$ and $\mathscr{P}_{2}$ of divisors on $Z^{\prime}$ are constructed. These play an important role in our proof of (i). Section 4 proves (i) of Main Theorem. Equivariant deformations of $Z^{\prime}$ are studied and it is proved that any element in $\mathscr{P}_{1}$ and $\mathscr{P}_{2}$ does not disappear under any $T^{2}$-equivariant deformation of $Z^{\prime}$. As a result, we complete our proof of (i). Section 5 determines relationship between the weighted dual graphs of the toric surfaces appeared as members of the pencil $|F|^{T^{2}}$ and those of $T^{2}$-actions on $n \mathrm{CP}^{2}$. This in particular implies (iv) of Main Theorem.

Recently, A. Fujiki [5] has proved a conjecture of D. Joyce [10, 3.3.4] which states that compact simply connected self-dual manifolds which admits an effective $T^{2}$-action must be conformally equivalent to Joyce's example [10]. $\mathrm{He}$ also described the structure of corresponding twistor space. In particular, Pedersen-Poon twistor spaces are nothing but the twistor spaces associated to the self-dual metrics of Joyce. As a result, our investigations here turned out to be equivalent to studying degenerations of the metrics of Joyce.

The author would like to thank A. Fujiki for many helpful conversations. He also would like to thank H. Pedersen and Y. S. Poon for kind encouragements.

\section{Preliminaries}

\subsection{Torus actions on $n \mathrm{CP}^{2}$}

In this subsection we first summarize results of Orlik and Raymond [14] and Joyce [10] about classification of torus actions on $n \mathrm{CP}^{2}$.

Let $\alpha: T^{2} \times n \mathrm{CP}^{2} \rightarrow n \mathrm{CP}^{2}$ be any effective action, $N$ the space of orbits and $\pi: M \rightarrow N$ the orbit map. Then $N$ is a closed 2-disk. Let $\partial N\left(\simeq S^{1}\right)$ be the boundary of $N$ and $N^{\circ}:=N \backslash \partial N$ the interior of $N$. Then the following (a)-(c) hold: 
(a) $\pi^{-1}\left(N^{\circ}\right)$ is isomorphic to $N^{\circ} \times T^{2} T^{2}$-equivariantly, where $T^{2}$ action on $N^{\circ} \times T^{2}$ is given by the product of the trivial action (on $N^{\circ}$ ) and the standard one (on itself),

(b) there exist distinguished $k:=n+2$ points $p_{1}, p_{2}, \ldots, p_{k}$ of $\partial N$ such that $\pi^{-1}\left(p_{i}\right)$ is a point for every $1 \leq i \leq k$. By renumbering we assume that $p_{1}, \ldots, p_{k}$ are arranged in the cyclic order of the negative (i.e., clockwise) direction,

(c) set $A_{i}:=\pi^{-1}\left(\left[p_{i}, p_{i+1}\right]\right)\left(\subseteq n \mathrm{CP}^{2}\right), 1 \leq i \leq k$, where $\left[p_{i}, p_{i+1}\right]$ denotes the connected interval in $\partial N$ with boundary $\left\{p_{i}, p_{i+1}\right\}$, and subscripts are counted modulo $k$. Then each $A_{i}$ is a $T^{2}$-invariant sphere on $n \mathrm{CP}^{2}$ and there exists a unique one-dimensional subgroup $G_{i}$ of $T^{2}$ such that $G_{i}$ acts trivially on $A_{i}$. ( $G_{i}$ is isomorphic to the circle.)

Once we fix a basis of $H_{1}\left(T^{2}, \mathrm{Z}\right) \simeq \mathrm{Z}^{2}, G_{i}$ is represented by a pair of coprime integer; that is, $G_{i}=\left\{(s, t) \in T^{2}=U(1) \times U(1) \mid s^{m_{i}} t^{n_{i}}=1\right\}$. Of course, $\left(m_{i}, n_{i}\right)$ is determined only up to sign.

Proposition 1.1 ([14], [10]). Let $\alpha: T^{2} \times n \mathrm{CP}^{2} \rightarrow n \mathrm{CP}^{2}$ be any effective action and $A_{1}, A_{2}, \ldots, A_{k} \subseteq n \mathrm{CP}^{2}(k=n+2)$ the set of $T^{2}$-invariant spheres as above, and $G_{i} \subseteq T^{2}(1 \leq i \leq k)$ the stabilizer subgroup of $A_{i}$ as above. Then there exists a basis of $H_{1}\left(T^{2}, Z\right)$ such that $G_{i}$ is represented by $\left(m_{i}, n_{i}\right)$ in the following form:

(i) $\left(m_{k}, n_{k}\right)=(1,0),\left(m_{1}, n_{1}\right)=(0,1)$,

(ii) $m_{i} \geq 1$ for any $i \geq 2$, and

(iii) $m_{i} n_{i+1}-m_{i+1} n_{i}=-1$ for any $1 \leq j \leq k-1$.

Conversely, any set of coprime integers $\left\{\left(m_{i}, n_{i}\right) \mid 1 \leq i \leq k\right\}$ satisfying (i)(iii) determines an effective $T^{2}$-action on $n \mathrm{CP}^{2}$ such that its stabilizer subgroups $\left\{G_{i}\right\}$ can be represented by $\left\{\left(m_{i}, n_{i}\right)\right\}$.
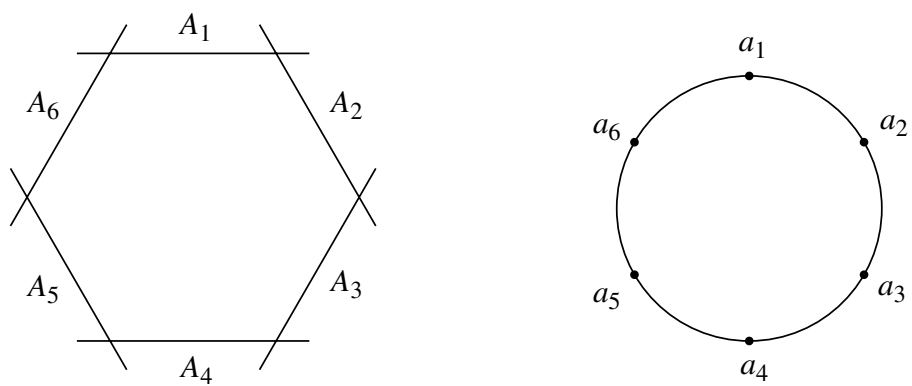

FiguRE 1

There is another representation of $T^{2}$-action on $n \mathrm{CP}^{2}$. Let $\alpha: T^{2} \times n \mathrm{CP}^{2} \rightarrow$ $n \mathrm{CP}^{2}$ be any effective action and $A:=\cup_{i=1}^{k} A_{i}$ the cycle of $T^{2}$-invariant spheres 
as above, and set $a_{i}:=A_{i}^{2}, 1 \leq i \leq k$, the self-intersection number of $A_{i}$ in $n \mathrm{CP}^{2}$. Then the weighted dual graph of $\alpha$ is the circle together with $k$ points $\beta_{1}, \ldots, \beta_{k}$ on the circle which are arranged in the cyclic order of the positive direction, and each $\beta_{i}$ is attached by the weight $a_{i}$.

Instead of this, we also denote $\left(a_{1}, a_{2}, \ldots, a_{k}\right)$ for short. The latter representation has an ambiguity of the permutations. Let $\alpha_{1}$ be the standard action on $\mathrm{CP}^{2}$ which is given by

$\alpha_{1}:(s, t) \times\left(\xi_{0}: \xi_{1}: \xi_{2}\right) \longmapsto\left(\xi_{0}: s \xi_{1}: t \xi_{2}\right), \quad(s, t) \in T^{2}=U(1) \times U(1)$,

where $\left(\xi_{0}: \xi_{1}: \xi_{2}\right)$ denotes a homogeneous coordinate on $\mathrm{CP}^{2}$. Then the dual graph of $\alpha_{1}$ is given by $(1,1,1)$.

The weighted dual graph also determines $T^{2}$-action on $n \mathrm{CP}^{2}$ : let $\alpha$ be any given effective $T^{2}$-action, $\left\{\left(m_{i}, n_{i}\right) \mid 1 \leq i \leq k\right\}$ the stabilizer data which is normalized as in Proposition 1.1, and $\left(a_{1}, a_{2}, \ldots, a_{k}\right)$ its weighted dual graph. Then a relation between these two representations is given as follows (subscripts are counted modulo $k$ ):

- $\left\{\left(m_{i}, n_{i}\right)\right\} \longmapsto\left(a_{i}\right)$ : each $a_{i}(1 \leq i \leq k)$ is given by ([14])

$$
a_{i}=-\left|\begin{array}{cc}
m_{i-1} & m_{i} \\
n_{i-1} & n_{i}
\end{array}\right|\left|\begin{array}{cc}
m_{i} & m_{i+1} \\
n_{i} & n_{i+1}
\end{array}\right|\left|\begin{array}{cc}
m_{i-1} & m_{i+1} \\
n_{i-1} & n_{i+1}
\end{array}\right| .
$$

(Note that the sign are different from $[14$, p. 544] because we choose the negative direction.)

- $\left(a_{i}\right) \longmapsto\left\{\left(m_{i}, n_{i}\right)\right\}$ : First set $\left(m_{k}, n_{k}\right)=(1,0)$ and $\left(m_{1}, n_{1}\right)=(0,1)$. Assume that $\left(m_{i-2}, n_{i-2}\right)$ and $\left(m_{i-1}, n_{i-1}\right)$ is determined for some $i \geq 1$. Then $\left(m_{i}, n_{i}\right)$ is determined as the unique solution of the following equations:

$$
\left|\begin{array}{cc}
m_{i-1} & m_{i} \\
n_{i-1} & n_{i}
\end{array}\right|=-1
$$

and

$$
a_{i-1}=-\left|\begin{array}{cc}
m_{i-2} & m_{i-1} \\
n_{i-2} & n_{i-1}
\end{array}\right|\left|\begin{array}{cc}
m_{i-1} & m_{i} \\
n_{i-1} & n_{i}
\end{array}\right|\left|\begin{array}{cc}
m_{i-2} & m_{i} \\
n_{i-2} & n_{i}
\end{array}\right| .
$$

(We have for example $\left(m_{2}, n_{2}\right)=\left(1, a_{1}\right),\left(m_{3}, n_{3}\right)=\left(a_{2}, a_{1} a_{2}-1\right),\left(m_{4}, n_{4}\right)=$ $\left(a_{2} a_{3}-1,-a_{1}-a_{3}+a_{1} a_{2} a_{3}\right), \ldots,\left(m_{k-1}, n_{k-1}\right)=\left(a_{k}, 1\right)$.) Consequently the weighted dual graph also determines $T^{2}$-action on $n \mathrm{CP}^{2}$. In the sequel we use this representation, rather than the stabilizer data. tion.

The dual graph of any effective $T^{2}$-action on $n \mathrm{CP}^{2}$ can by calculated by the following two propositions:

Proposition 1.2 (cf. [14, p. 553, Theorem]). Any effective $T^{2}$-action on $n \mathrm{CP}^{2}$ is obtained by a succession of $T^{2}$-equivariant connected sums of $\left(\mathrm{CP}^{2}, \alpha_{1}\right)$. 
Proposition 1.3. Let $\alpha: T^{2} \times n \mathrm{CP}^{2} \rightarrow \mathrm{CP}^{2}$ be any effective action and

$$
\left(a_{1}, a_{2}, \ldots, a_{k}\right)
$$

be the weighted dual graph of $\alpha$. Let $p:=A_{k} \cap A_{1}\left(=\pi^{-1}\left(p_{1}\right)\right) \in n \mathrm{CP}^{2}$ $\left(A_{i}=\pi^{-1}\left(\left[p_{i}, p_{i+1}\right]\right)\right.$ as above $)$ be a fixed point of $\alpha$. Let $q \in \mathrm{CP}^{2}$ be any fixed point of $\alpha_{1}$. Let $\alpha^{\prime}: T^{2} \times(n+1) \mathrm{CP}^{2} \rightarrow(n+1) \mathrm{CP}^{2}$ be the action obtained by $T^{2}$-equivariant connected sum of $\alpha$ and $\alpha_{1}$ at $p$ and $q$. Then the dual graph of $\alpha^{\prime}$ is given by

$$
\left(a_{1}+1, a_{2}, a_{3}, \ldots, a_{k-2}, a_{k-1}, a_{k}+1,1\right) .
$$

Proof. Recall that equivariant connected sum of $\alpha$ and $\alpha_{1}$ at $p$ and $q$ is made as follows: first choose $T^{2}$-invariant small neighborhood $U$ of $p$ and $V$ of $q=B_{3} \cap B_{1}$, where $B_{1}, B_{2}$ and $B_{3}$ are $\alpha_{1}$-invariant spheres in $\mathrm{CP}^{2}$. Next choose a $T^{2}$-equivariant, orientation reversing diffeomorphism $f: \partial U \rightarrow$ $\partial V$, where $\partial$ denotes the boundary. Put $s_{1}:=\partial U \cap A_{1}, s_{k}:=\partial U \cap A_{k}$, $t_{1}:=\partial V \cap B_{1}$ and $t_{3}:=\partial V \cap B_{3}$, all of which are $T^{2}$-invariant circles (if we choose $U$ and $V$ sufficiently small). Then equivariant connected sum is defined as $\left\{\left(n \mathrm{CP}^{2} \backslash U\right) \cup\left(\mathrm{CP}^{2} \backslash V\right)\right\} / \sim_{f}$, where $\sim_{f}$ means to identify $\partial U$ with $\partial V$ by $f$. Since $f$ is $T^{2}$-equivariant we have a natural $T^{2}$-action $\alpha^{\prime}$ on this space (which is $(n+1) \mathrm{CP}^{2}$ ).

The $T^{2}$-equivariance implies that either of the following holds:

$$
f\left(s_{1}\right)=t_{1}, \quad f\left(s_{k}\right)=t_{3}
$$

or

$$
f\left(s_{1}\right)=t_{3}, \quad f\left(s_{k}\right)=t_{1} .
$$

We only consider the former case because the latter can be treated in the same way. Let $A_{1}^{+} \subseteq A_{1}$ (resp. $B_{1}^{+} \subseteq B_{1}$ ) be the hemisphere which satisfies $p \notin A_{1}^{+}$ and $\partial A_{1}^{+}=s_{1}$ (resp. $q \notin B_{1}^{+}$and $\partial B_{1}^{+}=t_{1}$ ), and $A_{1}^{-} \subseteq A_{1}$ (resp. $B_{1}^{-} \subseteq B_{1}$ ) be the hemisphere which satisfies $p \in A_{1}^{-}$and $\partial A_{1}^{-}=s_{1}$ (resp. $q \in B_{1}^{-}$and $\partial B_{1}^{-}=t_{1}$ ). In a similar way, let $A_{k}^{+} \subseteq A_{k}$ (resp. $B_{3}^{+} \subseteq B_{3}$ ) be the hemisphere which satisfies $p \in A_{k}^{+}$and $\partial A_{k}^{+}=s_{k}$ (resp. $q \in B_{3}^{+}$and $\partial B_{3}^{+}=t_{3}$ ), and $A_{k}^{-} \subseteq A_{k}$ (resp. $B_{3}^{-} \subseteq B_{3}$ ) be the hemisphere which satisfies $p \notin A_{k}^{-}$ and $\partial A_{k}^{-}=s_{k}$ (resp. $q \notin B_{3}^{-}$and $\partial B_{3}^{-}=t_{3}$ ). Then we have $T^{2}$-invariant decompositions

$$
A_{1}=A_{1}^{+} \cup_{s_{1}} A_{1}^{-}, \quad A_{k}=A_{k}^{+} \cup_{s_{k}} A_{k}^{-}
$$

and

$$
B_{1}=B_{1}^{+} \underset{t_{1}}{\cup} B_{1}^{-}, \quad B_{3}=B_{3}^{+} \cup_{t_{3}}^{\cup} B_{3}^{-} .
$$


Then the new invariant spheres obtained by these procedures are

$$
A:=A_{1}^{+} \cup B_{1}^{+} \quad \text { and } \quad A^{\prime}:=A_{k}^{-} \cup B_{3}^{-} .
$$

We have only to show that $A^{2}=a_{1}+1$ and $\left(A^{\prime}\right)^{2}=a_{k}+1$. We put $p^{\prime}:=$ $A_{1} \cap A_{2}, p^{\prime \prime}:=A_{k-1} \cap A_{k}$ and $q^{\prime}:=B_{1} \cap B_{2}, q^{\prime \prime}:=B_{2} \cap B_{3}$. Let $u_{+}$(resp. $u_{-}$) be a complex coordinate on $A_{1} \backslash\{p\}$ (resp. $A_{1} \backslash\left\{p^{\prime}\right\}$ ) which satisfies $\left\{u_{+}=0\right\}=\left\{p^{\prime}\right\}$ (resp. $\left.\left\{u^{-}=0\right\}=\{p\}\right)$. We may assume that $u_{+} u_{-}=1$. In the same manner let $v_{+}$(resp. $v_{-}$) be a complex coordinate on $B_{1} \backslash\{q\}$ (resp. $B_{1} \backslash\left\{q^{\prime}\right\}$ ) which satisfies $\left\{v_{+}=0\right\}=\left\{q^{\prime}\right\}$ (resp. $\left\{v^{-}=0\right\}=\{q\}$ ) and $v_{+} v_{-}=1$. Choose a complex structure on the normal bundle $N_{A_{1} / n C \mathrm{P}^{2}}$ such that the complex orientation on the total space gives the reverse orientation to the standard one on $n \mathrm{CP}^{2}$. Then we choose a trivialization of $N_{A_{1} / n \mathrm{CP}^{2}}$ on $A_{1} \backslash\{p\}$ (resp. $A_{1} \backslash\left\{p^{\prime}\right\}$ ) and let $\xi_{+}$(resp. $\xi_{-}$) denote the induced fiber coordinate. Similarly, choose a complex structure and a trivialization of $N_{B_{1} / n C \mathrm{P}^{2}}$ on $B_{1} \backslash\{q\}$ (resp. $B_{1} \backslash\left\{q^{\prime}\right\}$ ) and let $\eta_{+}$(resp. $\eta_{-}$) denotes the induced fiber coordinate. Then $A_{1}^{2}=a_{1}$ and $B_{1}^{2}=1$ imply

$$
\xi_{-}=u_{+}^{a_{1}} \xi_{+}, \quad \eta_{-}=v_{+} \eta_{+} .
$$

On the other hand, since $f$ reverses the orientation and, the tangent spaces of $\partial U$ (resp. $\partial V$ ) are generated by the normal directions of $A_{1}$ (resp. $B_{1}$ ) in $n \mathrm{CP}^{2}$ (resp. $\mathrm{CP}^{2}$ ) and the tangent spaces of $s_{1}$ (resp. $t_{1}$ ), we may assume that $f$ gives an identification $u_{-}=v_{-}^{-1}$ and $\xi_{-}=\eta_{-}$on $s_{1} \simeq t_{1}$. As a consequence the relation between $\xi_{+}$and $\eta_{+}$is given by

$$
\xi_{+}=u_{+}^{-a_{1}} \xi_{-}=u_{-}^{a_{1}} \xi_{-}=u_{-}^{a_{1}} \eta_{-}=v_{-}^{-a_{1}} v_{+} \eta_{+}=v_{+}^{a_{1}+1} \eta_{+} .
$$

This implies that the degree of the normal bundle of $A$ in $(n+1) \mathrm{CP}^{2}$ is $a_{1}+1$. In the same way we can show that $\left(A^{\prime}\right)^{2}=a_{k}+1$, as claimed.

\subsection{Theory of toric varieties}

Let $N$ be a free Z-module of rank $r$ and put $N_{\mathrm{R}}:=N \otimes_{\mathrm{z}} \mathrm{R}, T_{N}:=N \otimes_{\mathrm{z}} \mathrm{C}^{*}$. A strongly convex rational polyhedral cone in $N_{\mathrm{R}}$ is a subset $\sigma \subseteq N_{\mathrm{R}}$ which can be expressed as $\sigma=\sum_{i=1}^{s} \mathrm{R}_{\geq 0} n_{i}$ for some $n_{1}, \ldots, n_{s} \in N$ and satisfies $\sigma \cap(-\sigma)=0$. We simply call such $\sigma$ a cone for short. Put $M:=\operatorname{Hom}_{\mathrm{Z}}(N, \mathrm{Z})$ and let $\langle\cdot, \cdot\rangle$ denotes the dual pairing. For a cone $\sigma$ in $N_{\mathrm{R}}$, let $\sigma^{\vee}:=\{x \in$ $M_{\mathrm{R}} \mid\langle x, y\rangle \geq 0$ for all $\left.y \in \sigma\right\}$ be the dual cone of $\sigma$ and put $S_{\sigma}:=M \cap \sigma^{\vee}$. Then by Gordan's lemma we can write $S_{\sigma}=\mathrm{Z}_{\geq 0} m_{1}+\cdots+\mathrm{Z}_{\geq 0} m_{p}$ for some $m_{1}, \ldots, m_{p} \in S_{\sigma}$. Further we set

$U_{\sigma}:=\left\{u: S_{\sigma} \rightarrow \mathrm{C} \mid u(0)=1, u\left(m+m^{\prime}\right)=u(m) u\left(m^{\prime}\right)\right.$ for any $\left.m, m^{\prime} \in S_{\sigma}\right\}$. 
Since $u \in U_{\sigma}$ is determined by $\left(u\left(m_{1}\right), \ldots, u\left(m_{p}\right)\right) \in \mathrm{C}^{p}$, and any (linear) relation $\sum_{i=1}^{p} a_{i} m_{i}=0\left(a_{i} \in \mathrm{Z}\right)$ changes into the relation $\prod_{i=1}^{p} u\left(m_{i}\right)^{a_{i}}=1$, we can regard $U_{\sigma}$ as an algebraic subset in $\mathrm{C}^{p}$ whose defining equations are of the form (monomial)=(monomial). In particular for $\sigma=0$, we have $U_{0}=$ $\left(C^{*}\right)^{r}$. Setting $(t u)(m):=t(m) u(m)$ for $t \in T_{N}, u \in U_{\sigma}$ and $m \in S_{\sigma}$, we get a natural $T_{N}$-action on $U_{\sigma}$. Further, we put $\sigma^{\perp}:=\left\{x \in M_{\mathrm{R}} \mid\langle x, y\rangle=0\right.$ for all $y \in \sigma\}$ and $\operatorname{orb}(\sigma):=\operatorname{Hom}_{\mathrm{Z}}\left(M \cap \sigma^{\perp}, \mathrm{C}^{*}\right)$. Assigning zero on the complement of $M \cap \sigma^{\perp}$, we have a natural embedding $\operatorname{orb}(\sigma) \hookrightarrow U_{\sigma}$. Then $\operatorname{orb}(\sigma)$ is a unique closed $T_{N}$-orbit in $U_{\sigma}$.

A cone $\tau$ in $N_{\mathrm{R}}$ is said to be a face of $\sigma$ and write $\tau \prec \sigma$ if there exists $m_{0} \in \sigma^{\vee}$ such that $\tau=\left\{y \in \sigma \mid\left\langle m_{0}, y\right\rangle=0\right\}$. In this case, we can naturally regard $U_{\tau}$ as a $T_{N}$-invariant open set in $U_{\sigma}$.

Definition 1.4. Let $\Delta:=\{\sigma\}$ be a collection of cones in $N_{\mathrm{R}} . \Delta$ is called a fan of $N$ if the following conditions are satisfied:

(i) if $\sigma \in \Delta$ then $\tau \in \Delta$ for any $\tau \prec \sigma$,

(ii) if $\sigma, \tau \in \Delta$ then $\sigma \cap \tau$ is a face of $\sigma$ and $\tau$.

Let $\Delta$ be a fan of $N$. Then by (ii) the set of affine algebraic varieties $\left\{U_{\sigma} \mid\right.$ $\sigma \in \Delta\}$ naturally patch together to obtain a variety $X:=X_{\Delta}:=\cup_{\sigma \in \Delta} U_{\sigma}$ on which $T_{N}$ acts naturally. $X_{\Delta}$ is called a toric variety associated to $\Delta$. We note that any fan contains $\sigma=0$ as a cone so that $X_{\Delta}$ always contains $U_{0} \simeq\left(\mathrm{C}^{*}\right)^{r}$ as a Zariski open set. Conversely, it is known that if an algebraic variety $X$ of dimension $r$ has an algebraic action of the algebraic torus $T_{\mathrm{C}}:=\left(\mathrm{C}^{*}\right)^{r}$ and if there exists an open orbit which is equivariantly isomorphic to $T_{\mathrm{C}}$, then there exists a fan $\Delta$ such that $X=X_{\Delta}$.

There are correspondences between properties of $\Delta$ and $X_{\Delta}$. For example, the compactness of $X_{\Delta}$ is equivalent to the completeness of $\Delta$; here, $\Delta$ is said to be complete if $|\Delta|=N_{R}$, where $|\Delta|$ denotes the support of $\Delta$ in $N_{\mathrm{R}} . X_{\Delta}$ is non-singular if and only if each $\sigma \in \Delta$ is non-singular in the following sense: there exists a basis $\left\{n_{1}, \ldots, n_{r}\right\}$ of $N$ and an integer $s \leq r$ such that $\sigma=\mathrm{R}_{\geq 0} n_{1}+\cdots+\mathrm{R}_{\geq 0} n_{s}$.

In what follows we only consider the case $r=2$. Let $\Delta \subseteq N_{\mathrm{R}}=\mathrm{R}^{2}$ be a complete non-singular fan and $X_{\Delta}$ the associated toric surface which is compact and non-singular. In this case, $X_{\Delta}$ can be described by another data instead of the fan: indeed, first by compactness, $\Delta$ is uniquely determined by the set of one-dimensional cones (i.e. half lines) $\left\{\sigma_{i}=\mathrm{R}_{\geq 0}\left(m_{i}, n_{i}\right)\right\}_{i=1}^{s}$. Here, we have fixed any basis of $N$ to represent the cones and $\left(m_{i}, n_{i}\right)$ is the pair of coprime integers. Second, the non-singularity implies that $\left|m_{i} n_{i+1}-m_{i+1} n_{i}\right|=$ 1 for any $1 \leq i \leq s$, where subscripts are counted modulo $s$. This implies that 
there uniquely exists a set of integers $\left\{a_{i}\right\}_{i=1}^{s}$ such that

$$
\left(m_{i-1}, n_{i-1}\right)+\left(m_{i+1}, n_{i+1}\right)+a_{i}\left(m_{i}, n_{i}\right)=0 \quad \text { for } \quad 1 \leq i \leq s .
$$

The weighted dual graph of $X_{\Delta}$ is defined by $\left(a_{1}, a_{2}, \ldots, a_{s}\right)$ : The meaning of this representation is the same as that of $\S 1.1$ except that the weighted points are arranged in the positive direction. It is easily verified that $a_{i}(1 \leq i \leq s)$ is explicitly given by

$$
a_{i}=-m_{i-1} n_{i+1}+m_{i+1} n_{i-1} .
$$

Finally, if $\left(a_{1}, a_{2}, \ldots, a_{s}\right)$ is the weighted dual graph of some compact nonsingular toric surface, we can recover a fan as follows: we first choose any $\left(m_{1}, n_{1}\right)$ and $\left(m_{2}, n_{2}\right)$ which satisfies $m_{1} n_{2}-m_{2} n_{1}=1$. Then $\left(m_{3}, n_{3}\right)$, $\left(m_{4}, n_{4}\right), \ldots\left(m_{s}, n_{s}\right)$ are determined inductively by (1) and the fan determined from $\left\{\left(m_{i}, n_{i}\right)\right\}_{i=1}^{r}$ is equivalent to the original fan under the $S L(2, \mathrm{Z})$-action. In consequence of these, it is equivalent for a non-singular toric surface to give a fan and to give the dual graph.

A geometric meaning of the weighted dual graph of $\Delta$ is similar to that on $\S 2.1$ : let $C_{i}(1 \leq i \leq s)$ be the closure of $\operatorname{orb}\left(\sigma_{i}\right)$ in $X_{\Delta} . C_{i}$ is a $T_{N}$-invariant non-singular rational curve. Then we have $C_{i}^{2}=a_{i}$.

In the subsequent sections we need the following two lemmas.

LEMMA 1.5. Let $(m, n)$ be a pair of coprime integer and $\sigma=\mathrm{R}_{\geq 0}(m, n) a$ one-dimensional cone in $\mathrm{R}^{2}$ and $U_{\sigma}$ the corresponding affine toric surface and $\operatorname{orb}(\sigma)$ the unique closed $T_{N}$-orbit in $U_{\sigma}$ as above. Then $U_{\sigma}$ is isomorphic to $\mathrm{C} \times \mathrm{C}^{*}$ and $\operatorname{orb}(\sigma)$ is given by $\{0\} \times \mathrm{C}^{*}$. Moreover the stabilizer subgroup of $\operatorname{orb}(\sigma)$ is given by

$$
\left\{(s, t) \in \mathrm{C}^{*} \times \mathrm{C}^{*} \mid s^{n} t^{-m}=1\right\} .
$$

LEMMA 1.6. Let $\Delta$ be a complete and non-singular fan in $N_{\mathrm{R}}=\mathrm{R}^{2}$ and $S$ the compact non-singular toric surface associated to $\Delta$. Let $H^{i}\left(-K_{S}\right)^{T_{N}}$ denote the linear subspace of $H^{i}\left(-K_{S}\right)$ generated by all elements which are $T_{N}$-fixed with respect to the natural $T_{N}$-action on $H^{i}\left(-K_{S}\right)$. Then we have

$$
H^{i}\left(-K_{S}\right)^{T_{N}} \simeq \begin{cases}\mathrm{C}, & i=0 \\ 0, & i \geq 1\end{cases}
$$

Proof. Since $S$ is rational, we have only to prove the claim for $i=0$ and 1. Let $\left\{\tau_{i}\right\}_{i=1}^{s}$ be the set of one-dimensional cones in $\Delta$ and $n_{i} \in \tau_{i} \cap N$ be the primitive point on $\tau_{i}$. Put $\sigma_{i}:=\tau_{i}+\tau_{i+1}$ for $1 \leq i \leq s$, where $\tau_{s+1}:=\tau_{1}$. 
Then $\left\{\sigma_{i}\right\}_{i=1}^{s}$ is the set of two-dimensional cones in $\Delta$. Let $h_{i}: \sigma_{i} \rightarrow \mathrm{R}$ be a function defined by

$$
h_{i}(x):=-x_{1}-x_{2},
$$

where $x_{1}$ and $x_{2}$ are real numbers which are uniquely determined from $x \in \sigma_{i}$ by the equation $x=x_{1} \tau_{i}+x_{2} \tau_{i+1}$ in $N_{\mathrm{R}}=\mathrm{R}^{2}$. Then $\left\{h_{i}\right\}_{i=1}^{s}$ naturally patch together to give a continuous function $h: N_{\mathrm{R}} \rightarrow \mathrm{R}$. Further we set

$$
W:=\left\{n \in N_{\mathrm{R}} \mid h(n) \leq 0\right\} .
$$

Then [13, Theorem 2.6] implies that

$$
H^{i}\left(S,-K_{S}\right)^{T_{N}} \simeq H_{W}^{i}\left(N_{\mathrm{R}}, \mathrm{C}\right),
$$

where the right-hand-side denotes the cohomology group of $N_{\mathrm{R}}$ with support $W$ and with coefficients in C. But since each $h_{i}$ is non-positive on $\sigma_{i}$, we have $W=N_{\mathrm{R}}$. Then the cohomology exact sequence

$$
\begin{aligned}
0 \rightarrow H_{W}^{0}\left(N_{\mathrm{R}}, \mathrm{C}\right) \rightarrow H^{0}\left(N_{\mathrm{R}}, \mathrm{C}\right) & \rightarrow H^{0}\left(N_{\mathrm{R}} \backslash W, \mathrm{C}\right) \\
& \rightarrow H_{W}^{1}\left(N_{\mathrm{R}}, \mathrm{C}\right) \rightarrow H^{1}\left(N_{\mathrm{R}}, \mathrm{C}\right)=0
\end{aligned}
$$

implies the claim.

\subsection{Pedersen-Poon twistor spaces}

In this subsection we recall a result of Pedersen and Poon [16] who proved the existence of twistor spaces over $n \mathrm{CP}^{2}$ on which $T^{2}$ acts. Their result relies on the theory of Donaldson and Friedman [3], which we first review briefly.

Let $\left(M_{1}, g_{1}\right)$ and $\left(M_{2}, g_{2}\right)$ be compact self-dual manifolds and $Z_{1}$ and $Z_{2}$ the associated twistor spaces respectively. $Z_{1}$ and $Z_{2}$ are three-dimensional compact complex manifolds with anti-holomorphic involutions, which we denote by $\sigma_{1}$ and $\sigma_{2}$ respectively. These are also called the real structures. Let $\pi_{i}: Z_{i} \rightarrow M_{i}(i=1,2)$ be the twistor fibrations. $\pi_{i}$ gives $Z_{i}$ a $\mathrm{CP}^{1}$ bundle structure over $M_{i}$, whose fibers are $\sigma_{i}$-invariant complex submanifolds of $Z_{i}$. Let $p_{1} \in M_{1}$ and $p_{2} \in M_{2}$ be any points and put $L_{i}:=\pi_{i}^{-1}\left(p_{i}\right)$. Let $\mu_{i}: Z_{i}^{\prime} \rightarrow Z_{i}$ be the blowing-up along $L_{i}, Q_{i}$ the exceptional divisor and $\sigma_{i}^{\prime}$ the real structure induced by $\sigma_{i}$. Since the normal bundle of $L_{i}$ in $Z_{i}$ is isomorphic to $H \oplus H$, where $H$ denotes the holomorphic line bundle over $L_{i}$ whose degree is one, $Q_{i}$ is biholomorphic to $\mathrm{CP}^{1} \times \mathrm{CP}^{1}$. The normal bundle of $Q_{i}$ in $Z_{i}^{\prime}$ is biholomorphic to $\mathscr{O}(-1,1)$, where we set $\mathscr{O}(0,1):=\mu_{i}^{*} \mathscr{O}_{L_{i}}(1)$.

Let $\rho: T_{p_{1}} M_{1} \rightarrow T_{p_{2}} M_{2}$ be an orientation reversing isometry. $\rho$ naturally induces a holomorphic identification between $Q_{1}$ and $Q_{2}$, which we also denote by $\rho$. $\rho$ preserves the real structures. Identifying $Q_{1}$ and $Q_{2}$ by $\rho$, we 
set

$$
Z^{\prime}:=Z_{1}^{\prime} \cup_{\rho} Z_{2}^{\prime}
$$

which can be regarded as a compact complex space that has only simple normal crossing singularities along $Q:=Q_{1} \simeq Q_{2}$. Let $\sigma^{\prime}$ be the real structure of $Z^{\prime}$ which is naturally induced by $\sigma_{1}^{\prime}$ and $\sigma_{2}^{\prime}$. Then since $\rho^{*} \mathscr{O}_{Q_{2}}(0,1) \simeq \mathscr{O}_{Q_{1}}(1,0)$, we have

$$
\Theta_{Z^{\prime}}^{1} \simeq N_{Q_{1} / Z_{1}^{\prime}} \otimes N_{Q_{2} / Z_{2}^{\prime}} \simeq \mathscr{O}_{Q}(-1,1) \otimes \mathscr{O}_{Q}(1,-1) \simeq \mathscr{O}_{Q}
$$

where we put $\Theta_{Z^{\prime}}^{1}:=\mathscr{E} x t_{\mathscr{O}_{Z^{\prime}}}^{1}\left(\Omega_{Z^{\prime}}, \mathscr{O}_{Z^{\prime}}\right)\left(\Omega_{Z^{\prime}}\right.$ is the sheaf of Kähler differential of $Z^{\prime}$; see $\S 4$ for the definition) and $N_{Q_{i} / Z_{i}^{\prime}}$ is the normal bundle of $Q_{i}$ in $Z_{i}^{\prime}$. Hence $Z^{\prime}$ is $d$-semi-stable in the sense of Friedman [4]. Then one of the results of [3] is the following

Proposition 1.7 ([3]). Suppose that $H^{2}\left(Z_{1}, \Theta_{Z_{1}}\right)=H^{2}\left(Z_{2}, \Theta_{Z_{2}}\right)=0$. Then there exist

- non-singular complex space $\mathscr{Z}$ with an anti-holomorphic involution $\sigma$, and

- a proper surjective holomophic map $p: \mathscr{Z} \rightarrow \Delta$, where $\Delta$ is a disk in $\mathrm{C}$ which is invariant under the complex conjugation $\sigma$, such (i)-(iv) below are fulfilled:

(i) There exists a biholomorphic map between $Z^{\prime}$ and $p^{-1}(0)$ preserving the real structures,

(ii) $p$ is smooth (i.e. maximal rank) on $\mathscr{Z} \backslash p^{-1}(0)$,

(iii) $p \cdot \sigma=\underline{\sigma} \cdot p$, and if $t$ is non-zero and real with respect to $\underline{\sigma}$, then $Z_{t}:=p^{-1}(t)$ is a twistor space over $M_{1} \# M_{2}$,

(iv) $H^{2}\left(Z_{t}, \Theta_{Z_{t}}\right)=0$ for any $t \in \Delta \backslash\{0\}$.

As a consequence of this theorem, Donaldson and Friedman proved the existence of twistor spaces on $n \mathrm{CP}^{2}$ in the following way. First set $M_{1}=$ $M_{2}=\mathrm{CP}^{2}$ with the Fubini-Study metric. The corresponding twistor spaces $Z_{i}$ $(i=1,2)$ are a flag manifold $\mathrm{F}$ (cf. Example 2.4), which satisfies $H^{2}\left(\Theta_{\mathrm{F}}\right)=0$. Hence we may apply Proposition 1.7 to obtain a twistor space $Z$ over $2 \mathrm{CP}^{2}$ which satisfies $H^{2}\left(\Theta_{Z}\right)=0$. (This is nothing but the twistor space discovered by Poon [19].) Next set $Z_{1}:=Z$ and $Z_{2}:=\mathrm{F}$ and apply Proposition 1.7 again. Then we get twistor space over $3 C P^{2}$ and it satisfies $H^{2}(\Theta)=0$. Repeating this procedure, they inductively obtain a twistor space over $n \mathrm{CP}^{2}$ for any $n \geq 1$.

Next let us explain an equivariant version of this result, which is due to Pedersen and Poon [16]. Let $\left(M_{1}, g_{1}\right),\left(M_{2}, g_{2}\right), Z_{1}, Z_{2}, \pi_{1}, \pi_{2}, \sigma_{1}$ and $\sigma_{2}$ be as above, and $G$ a compact Lie group acting isometrically both on $\left(M_{1}, g_{1}\right)$ and $\left(M_{2}, g_{2}\right)$. We assume that there exist $G$-fixed points $p_{1} \in M_{1}$ and $p_{2} \in M_{2}$ such 
that there exists an orientation reversing isometry $\rho: T_{p_{1}} M_{1} \rightarrow T_{p_{2}} M_{2}$ which is $G$-equivariant with respect to the isotropic representations. This condition implies that we can make $G$-equivariant connected sum of $M_{1}$ and $M_{2}$ at $p_{i}$. The $G$-action on $M_{i}$ naturally gives rise to a holomorphic $G$-action on $Z_{i}$. Then since $L_{i}=\pi^{-1}\left(p_{i}\right)(i=1,2)$ is $G$-invariant, $Z_{i}^{\prime}$ also has a holomorphic $G$ action with respect to which $Q_{i}$ is invariant. Further, because $\rho: Q_{1} \rightarrow Q_{2}$ is $G$-equivariant, the complex space $Z^{\prime}:=Z_{1}^{\prime} \cup_{\rho} Z_{2}^{\prime}$ also has a $G$-action.

Proposition 1.8 ([16]). Suppose that $H^{2}\left(Z_{1}, \Theta_{Z_{1}}\right)=H^{2}\left(Z_{2}, \Theta_{Z_{2}}\right)=0$. Then there exists a $G$-equivariant deformation $Z_{t}$ of $Z^{\prime}$ such that $Z_{t}$ is a twistor space over $M_{1} \# M_{2}$ which satisfies $H^{2}\left(Z_{t}, \Theta_{Z_{t}}\right)=0$. In particular, $M_{1} \# M_{2}$ admits a self-dual metric with a $G$-symmetry.

This result implies that if the above machinery of Donaldson-Friedman can be applied for $Z_{1} \rightarrow M_{1}$ and $Z_{2} \rightarrow M_{2}$ to prove the existence of twistor space over $M_{1} \# M_{2}$, then its equivariant version automatically works so long as one can take equivariant connected sum of $M_{1}$ and $M_{2}$.

Let $\alpha: T^{2} \times n \mathrm{CP}^{2} \rightarrow n \mathrm{CP}^{2}$ be any effective $T^{2}$-action. Then by Proposition $1.2 \alpha$ is obtained as a succession of $T^{2}$-equivariant connected sums of $\mathrm{CP}^{2}$ with the standard action $\alpha_{1}$ (see $\S 2.1$ ). Hence by applying Proposition 1.8 $n$ times, we get a twistor space over $n \mathrm{CP}^{2}$ which has a holomorphic $T^{2}$-action. By construction, twistor fibration $\pi: Z \rightarrow n \mathrm{CP}^{2}$ is $T^{2}$-equivariant.

Definition 1.9. We call this twistor space a Pedersen-Poon twistor space associated to $\alpha$.

\section{Reducible members of the pencil $|F|^{T^{2}}$}

In this section we show (ii) and (iii) of Main Theorem follow from that of (i).

Let $\alpha: T^{2} \times n \mathrm{CP}^{2} \rightarrow n \mathrm{CP}^{2}$ be any effective $T^{2}$-action and $g$ any selfdual metric on $n \mathrm{CP}^{2}$, with respect to which $\alpha$ is an isometric action. Let $Z$ be the twistor space of $(M, g)$ and $\sigma$ the real structure of $Z$. (We do not assume here that $Z$ is a Pedersen-Poon twistor space.) Recall that there exists a holomorphic line bundle $F$ over $Z$ which is characterized by $F \otimes F \simeq-K_{Z}$ (cf. Introduction). Let $|F|^{T^{2}}$ denote the linear subsystem of $|F|$ associated to $H^{0}(Z, F)^{T^{2}}$, where $H^{0}(Z, F)^{T^{2}}$ denotes the linear subspace of $H^{0}(Z, F)$ generated by all $T^{2}$-fixed sections.

To clarify the reasoning of Main Theorem, we introduce the following

Definition 2.1. $Z$ is said to satisfy Condition $(A)$ if $Z$ satisfies (i) of Main Theorem. That is, $|F|^{T^{2}}$ is one-dimensional as a linear system and their generic members are biholomorphic to a non-singular toric surface.

Remark 2.2. Let $\left(n \mathrm{CP}^{2}, g\right), \alpha$ and $Z$ be as above assume that $Z$ satisfies Condition (A). Then the type of scalar curvature of the conformal class $[g]$ 
is positive. In fact, Condition (A) implies $a(Z) \geq 1$, where $a(Z)$ denotes the algebraic dimension of $Z$. Therefore results of Pontecorvo [18, 3.5, 3.3, 4.4] together with the simply connectedness of $n \mathrm{CP}^{2}$ show the claim. In particular, we may use the vanishing theorem of Hitchin [6].

EXAmple 2.3. The twistor space of the Euclidean four-sphere $S^{4}$ is $C P^{3}$, the three-dimensional complex projective space. It is convenient to regard $S^{4}$ as the quaternionic projective line $\mathrm{HP}^{1}$. Let $\left(q_{0}: q_{1}\right)$ be a homogeneous coordinate on $\mathrm{HP}^{1}$ and $\alpha_{0}$ be a $T^{2}$-action on $\mathrm{HP}^{1}$ given by

$$
\left(q_{0}: q_{1}\right) \stackrel{(s, t)}{\longmapsto}\left(s q_{0}: t q_{1}\right), \quad(s, t) \in T^{2}=U(1) \times U(1) .
$$

The fixed locus of $\alpha_{0}$ is $\{(1: 0),(0: 1)\}$ and the dual graph is given by $(0,0)$. We can explicitly see that the twistor space $C P^{3}$ satisfies Condition (A) as follows.

Let $\left(z_{0}: z_{1}: z_{2}: z_{3}\right)$ be a homogeneous coordinate on $\mathrm{CP}^{3}$. The real structure $\sigma$ and the natural lifting of $\alpha_{0}$ on $\mathrm{CP}^{3}$ is respectively given by

$$
\left(z_{0}: z_{1}: z_{2}: z_{3}\right) \stackrel{\sigma}{\longmapsto}\left(\bar{z}_{1}:-\bar{z}_{0}: \bar{z}_{3}:-\bar{z}_{2}\right)
$$

and

$$
\left(z_{0}: z_{1}: z_{2}: z_{3}\right) \stackrel{(s, t)}{\longmapsto}\left(s z_{0}: s^{-1} z_{1}: t z_{2}: t^{-1} z_{3}\right) .
$$

Since $K_{\mathrm{CP}^{3}}=\mathscr{O}_{\mathrm{CP}^{3}}(-4)$, we have $F=-\frac{1}{2} K_{\mathrm{CP}^{3}}=\mathscr{O}_{\mathrm{CP}^{3}}(2)$. Hence we get

$$
H^{0}(Z, F)^{T^{2}}=\left\langle z_{0} z_{1}, z_{2} z_{3}\right\rangle .
$$

As a consequence the reducible members of $|F|^{T^{2}}$ are $\left\{z_{0} z_{1}=0\right\}$ and $\left\{z_{2} z_{3}=\right.$ $0\}$ and all of the other members are non-singular toric surfaces biholomorphic to $\mathrm{CP}^{1} \times \mathrm{CP}^{1}$. Thus we have checked Condition (A).

The next example will be used to prove Main Theorem.

EXAMPLE 2.4. The twistor space of $C \mathrm{P}^{2}$ with the Fubini-Study metric is a flag manifold which is defined by

$$
\mathrm{F}:=\left\{(x, l) \in \mathrm{CP}^{2} \times\left(\mathrm{CP}^{2}\right)^{*} \mid x \in l\right\},
$$

where $\left(\mathrm{CP}^{2}\right)^{*}$ denotes the dual projective plane. Let $\left(z_{0}: z_{1}: z_{2}\right)$ and $\left(w_{0}\right.$ : $\left.w_{1}: w_{2}\right)$ be homogeneous coordinates on $\mathrm{CP}^{2}$ and $\left(\mathrm{CP}^{2}\right)^{*}$ respectively so that $F$ is expressed by an equation

$$
z_{0} w_{0}+z_{1} w_{1}+z_{2} w_{2}=0 .
$$


The real structure $\sigma$ and the natural lifting of $\alpha_{1}$ (cf. §1.1) on F, are respectively given by

$$
\left(z_{0}: z_{1}: z_{2}\right) \times\left(w_{0}: w_{1}: w_{2}\right) \stackrel{\sigma}{\longmapsto}\left(\bar{w}_{0}: \bar{w}_{1}: \bar{w}_{2}\right) \times\left(\bar{z}_{0}: \bar{z}_{1}: \bar{z}_{2}\right)
$$

and

$$
\left(z_{0}: z_{1}: z_{2}\right) \times\left(w_{0}: w_{1}: w_{2}\right) \stackrel{(s, t)}{\longmapsto}\left(z_{0}: s z_{1}: t z_{2}\right) \times\left(w_{0}: s^{-1} w_{1}: t^{-1} w_{2}\right) .
$$

By adjunction formula, we have $F=\mathscr{O}_{\mathrm{F}}(1,1)\left(:=\left.\mathscr{O}_{\mathrm{CP}^{2} \times\left(\mathrm{CP}^{2}\right)^{*}}(1,1)\right|_{\mathrm{F}}\right)$. It is easily verified that

$$
H^{0}(\mathrm{~F}, F)^{T^{2}}=\left\langle z_{0} w_{0}, z_{1} w_{1}, z_{2} w_{2}\right\rangle
$$

and the reducible members are $\left\{z_{0} w_{0}=0\right\},\left\{z_{1} w_{1}=0\right\}$ and $\left\{z_{2} w_{2}=0\right\}$. (Note that the system $\left\langle z_{0} w_{0}, z_{1} w_{1}, z_{2} w_{2}\right\rangle$ is one-dimensional as a linear system because the equation (3) holds on $F$ ). From these, we can easily see that $F$ together with $\alpha_{1}$ satisfies Condition (A). We note that $H^{2}\left(\mathrm{~F}, \Theta_{\mathrm{F}} \otimes F^{-1}\right)=0$ as is easily shown by Akizuki-Nakano vanishing (see the proof of [7, Lemma 4.2]).

We recall the following three results which are needed to study the reducible members of $|F|^{T^{2}}$.

Proposition 2.5 ([20]). Let $Z$ be any twistor space over $n \mathrm{CP}^{2}$ and $D$ any divisor on $Z$ which satisfies $D \cdot L^{\prime}=1$, where $L^{\prime}$ is a twistor line on $Z$. Then $D$ is non-singular and obtained from $\mathrm{CP}^{2}$ by blowing-up n points. Further $D \cap \bar{D}$ coincides with a twistor line $L$ and the intersection is transversal. Moreover, the first Chern class of the line bundle $[D]$ satisfies

$$
c_{1}([D])=\frac{1}{4} c_{1}(Z)+\frac{1}{2} \sum_{i=1}^{n} \sigma_{i} \pi^{*} \xi_{i}
$$

for some orthonormal basis $\left\{\xi_{1}, \ldots, \xi_{n}\right\}$ of $H^{2}\left(n \mathrm{CP}^{2}, \mathrm{Z}\right)$ and $\sigma_{i}$ equal to 1 or -1 .

Proposition 2.6 ([15]). Let $Z$ be any twistor space over $n \mathrm{CP}^{2}$. For any real and irreducible member $S$ of $|F|$, there exists a birational morphism $\mu$ : $S \rightarrow \mathrm{CP}^{1} \times \mathrm{CP}^{1}$ which preserves the real structure. Moreover $c_{1}^{2}(S)=8-2 n$ and the resultant real structure of $\mathrm{CP}^{1} \times \mathrm{CP}^{1}$ is given by (anti-podal map) $\times$ (complex conjugation). Furthermore the blown-up points of $\mu$ never lie on $\mathrm{CP}^{1} \times($ the real circle $)$.

Let $S \in|F|$ and $\mu: S \rightarrow \mathrm{CP}^{1} \times \mathrm{CP}^{1}$ be as in the above proposition. $\mu$ naturally defines the exceptional curves $E_{1}, \ldots, E_{n}$ which satisfy $E_{i} \cdot E_{j}=$ 
$\bar{E}_{i} \cdot \bar{E}_{j}=-\delta_{i j}$ and $E_{i} \cdot \bar{E}_{j}=0$ for any $1 \leq i, j \leq n$. Let $\left\{\xi_{1}, \ldots, \xi_{n}\right\}$ be the orthonormal basis of $H^{2}\left(n \mathrm{CP}^{2}, \mathrm{Z}\right)$ which is naturally determined by the condition

$$
\left.\pi^{*} \xi_{i}\right|_{S}=E_{i}-\bar{E}_{i} \quad \text { for } \quad 1 \leq i \leq n .
$$

(This is possible because $\left.\pi\right|_{S}: S \rightarrow n C P^{2}$ is a ramified double covering and the blown-up points of $\mu$ do not lie on the ramification locus $\mathrm{CP}^{1} \times$ (the real circle) [15]). Put $\alpha_{i}:=\pi^{*} \xi_{i} \in H^{2}(Z, Z)(1 \leq i \leq n)$. Let $\sigma_{i}(1 \leq i \leq n)$ be integers which are equal to 1 or -1 and $\mathscr{D}_{\sigma_{1} \cdots \sigma_{n}}$ the holomorphic line bundle over $Z$ whose first Chern class is $\frac{1}{4} c_{1}(Z)+\frac{1}{2} \sum_{i=1}^{n} \sigma_{i} \alpha_{i} \in H^{2}(Z, Z)$. (Here we have used the vanishing theorem of Hitchin to see that $H^{2}(Z, Z) \simeq$ Pic $Z$ ).

Proposition 2.7 ([7]). As elements of $H^{2}(S, Z)$, we have

$$
\left.\mathscr{D}_{\sigma_{1} \cdots \sigma_{n}}\right|_{S}=\mu^{*} \mathscr{O}(1,1)-\frac{1}{2} \sum_{i=1}^{n}\left(1-\sigma_{i}\right) E_{i}-\frac{1}{2} \sum_{i=1}^{n}\left(1+\sigma_{i}\right) \bar{E}_{i} .
$$

Let $Z$ be a twistor space over $n \mathrm{CP}^{2}$ which satisfies Condition (A) and $S \in$ $|F|^{T^{2}}$ a generic member which is an irreducible non-singular toric surface. In addition we assume that $S$ is real. (Practically it suffices to suppose that $S$ is real and reducible. See Remark 2.9.) Since $c_{1}^{2}(S)=8-2 n$, the number of one-dimensional orbits of the $\left(C^{*}\right)^{2}$-action is $2 n+4$. Let $\left\{C_{i}\right\}_{i=1}^{2 n+4}$ be the set of one-dimensional orbit. Then it is well known that $C:=\sum_{i=1}^{2 n+4} C_{i}$ is the only $\left(C^{*}\right)^{2}$-invariant anticanonical curve of $S$ and that by renumbering if necessary we may assume that

$$
C_{i} \cdot C_{j}= \begin{cases}1, & |i-j|=1,2 n+3, \\ 0, & |i-j| \geq 2 .\end{cases}
$$

Since $S$ is real, $C$ is also real. Then Proposition 2.6 implies that $\bar{C}_{i} \neq C_{i}$ for any $i$, because the blown-up points of $\mu$, which must lie on the $T^{2}$ fixed points, never lie on the real locus. So we may write $C=\sum_{i=1}^{k}\left(C_{i}+\bar{C}_{i}\right)$, where we set $k:=n+2$. Then we put $q_{i}:=C_{i-1} \cap C_{i}$ for $2 \leq i \leq k$ and $q_{1}:=C_{k} \cap C_{1}$. Then $\left\{q_{i}, \bar{q}_{i} \mid 1 \leq i \leq k\right\}$ is the set of $T^{2}$-fixed points of $S$. Let $L_{i}(1 \leq i \leq k)$ be the twistor line which goes through $q_{i}$ and $\bar{q}_{i}$. For each $i, 1 \leq i \leq k$, we decompose $C$ as follows: $C=C_{(i)}+\bar{C}_{(i)}$, where $C_{(i)}$ and $\bar{C}_{(i)}$ are connected curves satisfying $C_{(i)} \cap \bar{C}_{(i)}=\left\{q_{i}, \bar{q}_{i}\right\}$.

Proposition 2.8 (See also [5]). Let $Z$ be a twistor space over $n \mathrm{CP}^{2}$ with $T^{2}$-action which satisfies Condition (A) and $S, C, C_{i}, C_{(i)}$ and $L_{i}$ as above. Then

(i) the base locus of $|F|^{T^{2}}$ coincides with $C$, 
(ii) the number of reducible members of $|F|^{T^{2}}$ is $k=n+2$, all of which are of the form $D_{i}+\bar{D}_{i}(1 \leq i \leq k)$, where every $D_{i}(1 \leq i \leq k)$ is an irreducible non-singular toric surface,

(iii) for each $D_{i}$ in (ii), there exists $j, 1 \leq j \leq k$ such that $D_{i} \cap S=C_{(j)}$ (and $\bar{D}_{i} \cap S=\bar{C}_{(j)}$ ),

(iv) renumbering $\left\{D_{j}\right\}$ if necessary we assume that $D_{i} \cap S=C_{(i)}$ for any $1 \leq i \leq k$. Then $D_{i} \cap \bar{D}_{i}=L_{i}$ for any $1 \leq i \leq k$,

(v) any irreducible non-singular member of $|F|^{T^{2}}$ does not contain $L_{i}$ for any $1 \leq i \leq k$.

In particular Condition (A) implies (ii) and (iii) of Main Theorem.

Proof of Proposition 2.8. (i) Let $S \in|F|^{T^{2}}$ be as proposition (that is, $S$ is non-singular and real member). Then since $\left.F\right|_{S} \simeq-K_{S}$ by adjunction, we have a $T^{2}$-equivariant exact sequence

$$
0 \longrightarrow \mathscr{O}_{Z} \longrightarrow F \longrightarrow-K_{S} \longrightarrow 0 \text {. }
$$

By the simply connectedness, we have $H^{1}\left(Z, \mathscr{O}_{Z}\right)=0$ ([12]). Hence we obtain $T^{2}$-equivariant exact sequence of the cohomology groups:

$$
0 \longrightarrow H^{0}\left(Z, \mathscr{O}_{Z}\right) \longrightarrow H^{0}(Z, F) \longrightarrow H^{0}\left(S,-K_{S}\right) \longrightarrow 0 .
$$

Since $T^{2}$ is a compact group, the $T^{2}$-fixed parts of each term yields the exact sequence

$$
0 \longrightarrow H^{0}\left(Z, \mathscr{O}_{Z}\right)^{T^{2}} \longrightarrow H^{0}(Z, F)^{T^{2}} \longrightarrow H^{0}\left(S,-K_{S}\right)^{T^{2}} \longrightarrow 0 .
$$

Lemma 1.6 implies that $C$ is the zero locus of a non-zero element of $H^{0}\left(S,-K_{S}\right) \simeq \mathrm{C}$. Hence (4) implies that Bs $|F|^{T^{2}}=\mathrm{Bs}\left|-K_{S}\right|^{T^{2}}=\{C\}$.

(ii),(iii) Let $D+D^{\prime}$ be any reducible member of $|F|^{T^{2}}$. First we show that $\bar{D}=D^{\prime}$. By Proposition 2.5 we have $c_{1}([\bar{D}])=c_{1}\left(\left[D^{\prime}\right]\right)$, where $[\bar{D}]$ and $\left[D^{\prime}\right]$ denote the line bundles determined by $\bar{D}$ and $D^{\prime}$ respectively. Hence $D^{\prime} \neq \bar{D}$ implies that $D^{\prime}$ and $\bar{D}$ are distinct members of the same linear system (because we have $H^{2}(Z, Z) \simeq$ Pic $Z$ by the exponential sequence and the vanishing theorem of Hitchin). This implies [20, Theorem 3.1] that $Z$ is a twistor space of LeBrun (with $T^{2}$-action) ([11]). Let $\Phi: Z \rightarrow Q:=\mathrm{CP}^{1} \times \mathrm{CP}^{1} \subseteq \mathrm{CP}^{3}$ be the rational map associated to the system $|F|$, which is three dimensional. Then the pencil $|F|^{T^{2}}$ is the pull-back of certain pencil in $\left|\mathscr{O}_{Q}(1,1)\right|$ and we can explicitly check that any reducible member of $|F|^{T^{2}}$ is real, because we have an explicit defining equation of these twistor spaces. Thus even in the LeBrun case, any reducible member of $|F|^{T^{2}}$ is real.

Next we show that there do exist $k=n+2$ reducible members of $|F|^{T^{2}}$ and that they satisfy (iii). We use the argument in the proof of [7, Proposition 1.2]. 
For $\sigma_{1}, \ldots, \sigma_{n}$ with all $\sigma_{i}$ equal to 1 or $-1, \mathscr{D}_{\sigma_{1} \ldots \sigma_{n}}$ denotes the holomorphic line bundle over $Z$ as before. We consider an exact sequence

$$
\left.0 \longrightarrow \mathscr{D}_{\sigma_{1} \cdots \sigma_{n}} \otimes F^{-1} \longrightarrow \mathscr{D}_{\sigma_{1} \cdots \sigma_{n}} \longrightarrow \mathscr{D}_{\sigma_{1} \cdots \sigma_{n}}\right|_{S} \longrightarrow 0
$$

Note that this is a $T^{2}$-equivariant sequence, since $H^{2}(Z, Z) \simeq \operatorname{Pic} Z$ as we have seen above. Now as shown in the proof of [7, Proposition 1.2], we have $H^{i}\left(\mathscr{D}_{\sigma_{1} \cdots \sigma_{n}} \otimes F^{-1}\right)=0$ for any $i \geq 0$ and hence (5) in particular induces a $T^{2}$-equivariant isomorphism

$$
H^{0}\left(Z, \mathscr{D}_{\sigma_{1} \cdots \sigma_{n}}\right) \simeq H^{0}\left(S, \mathscr{D}_{\sigma_{1} \cdots \sigma_{n}} \mid S\right)
$$

We now show that for any $i, 1 \leq i \leq k$, there exists a set $\left\{\sigma_{1}, \ldots, \sigma_{n} \mid \sigma_{i}= \pm 1\right\}$ such that $C_{(i)}$ is a member of $\left|\mathscr{D}_{\sigma_{1} \cdots \sigma_{n}}\right| S \mid$. We may write

$$
C_{(i)}=\mu^{*} \mathscr{O}(\alpha, \beta)-\sum_{i=1}^{n} a_{i} E_{i}-\sum_{i=1}^{n} b_{i} \bar{E}_{i}
$$

for some integers $\alpha, \beta, a_{i}$ and $b_{i}$. But since $C_{(i)}+\bar{C}_{(i)}=C \in\left|-K_{S}\right|$, we have

$$
\alpha=\beta=1 \quad \text { and } \quad a_{i}+b_{i}=1
$$

for any $1 \leq i \leq n$. Hence we have

$$
C_{(i)}^{2}=2-\sum_{i=1}^{n} a_{i}^{2}-\sum_{i=1}^{n} b_{i}^{2}=2-n-2 \sum_{i=1}^{n} a_{i}^{2}+2 \sum_{i=1}^{n} a_{i}
$$

On the other hand, we have

$8-2 n=c_{1}^{2}(S)=C^{2}=\left(C_{(i)}+\bar{C}_{(i)}\right)^{2}=C_{(i)}^{2}+\bar{C}_{(i)}^{2}+2 C_{(i)} \cdot \bar{C}_{(i)}=2 C_{(i)}^{2}+4$.

Therefore we get

$$
C_{(i)}^{2}=2-n .
$$

Combining (7) and (8), we get $\sum_{i=1}^{n} a_{i}^{2}=\sum_{i=1}^{n} a_{i}$. This implies that every $a_{i}$, is equal to 1 or 0 . Let $I \subseteq\{1,2, \ldots, n\}$ (resp. $J \subseteq\{1,2, \ldots, n\}$ be the subset such that $i \in I$ (resp. $i \in J$ ) implies $a_{i}=1$ (resp. $a_{i}=-1$ ). We set $\sigma_{i}=-1$ for $i \in I$ and $\sigma_{i}=1$ for $i \in J$. Then we have

$$
C_{i}=\mu^{*} \mathscr{O}(1,1)-\frac{1}{2} \sum_{i=1}^{n}\left(1-\sigma_{i}\right) E_{i}-\frac{1}{2} \sum_{i=1}^{n}\left(1+\sigma_{i}\right) \bar{E}_{i} .
$$


Hence by Proposition 2.7, $C_{(i)}$ is a member of $\left|\mathscr{D}_{\sigma_{1} \cdots \sigma_{n}}\right| S \mid$. Further by (6) $C_{(i)}$ uniquely extends to $T^{2}$-invariant divisor $D_{i} \in\left|\mathscr{D}_{\sigma_{1} \cdots \sigma_{n}}\right|$. Thus we have seen that for each $i$ there exist $\sigma_{1}, \ldots, \sigma_{n}(= \pm 1)$ such that $D_{i} \in\left|\mathscr{D}_{\sigma_{1} \cdots \sigma_{n}}\right|$ and it satisfies $D_{i} \cap S=C_{(i)}$.

Next we show that any reducible member of $|F|^{T^{2}}$ must coincide with one of the above $D_{i}+\bar{D}_{i}$. Any reducible member $D+\bar{D}$ of $|F|^{T^{2}}$ determines a decomposition $C=A+\bar{A}$, where $A=D \cap S$ and $\bar{D}=\bar{A} \cap S$. It suffices to show that $A$ is connected. Let $m \geq 1$ be the number of connected component of $A$. Then in the same manner as in (8), we have $A^{2}=2-n$. Hence we have $(A+\bar{A})^{2}=A^{2}+\bar{A}^{2}+2 A \cdot \bar{A}=2(2-n)+4 m$. On the other hand we have $(A+\bar{A})^{2}=8-2 n$. From these, we get $m=1$, which implies that $A$ is connected.

(iv) We may assume that $D_{i} \cap S=C_{(i)}$. Set $L:=D_{i} \cap \bar{D}_{i}$, which is a twistor line by Proposition 2.5. Since both $D_{i}$ and $\bar{D}_{i}$ are $T^{2}$-invariant, $L$ is also $T^{2}$-invariant. This implies that the point $p:=\pi(L) \in n \mathrm{CP}^{2}$ is a $T^{2}$-fixed point. But since $q_{i}$ (and $\bar{q}_{i}$ ) lies on $L$, we have $p=p_{i}$. That is $L=L_{i}$.

(v) Let $S \in|F|^{T^{2}}$ be a generic non-singular member which is not necessarily real. Then we also have $c_{1}^{2}(S)=8-2 n$, which implies that the number of onedimensional orbits of $\left(C^{*}\right)^{2}$-action is $2 k$. By (i) $S$ contains $C=\sum_{i=1}^{k}\left(C_{i}+\bar{C}_{i}\right)$ and hence cannot contain $L_{j}$ which is also $\left(C^{*}\right)^{2}$-invariant orbit.

REMARK 2.9. We can show the following: Let $Z$ be a twistor space over $n \mathrm{CP}^{2}$ with $T^{2}$-action and $S \in|F|$ be a real irreducible $T^{2}$-invariant member. Then $S$ is a non-singular toric surface. Proof: Proposition 2.6 directly implies the non-singularity of such $S$. Let $\left(\mathrm{C}^{*}\right)^{2} \times S \rightarrow S$ be the complexification of the $T^{2}$-action. Then since $S$ being algebraic, the orbit which goes through generic point of $S$ must $\left(C^{*}\right)^{2}$ itself. In fact, let $s$ be any point on two-dimensional orbit of the $\left(C^{*}\right)^{2}$-action and assume that $s$ has non-trivial stabilizer $g \in\left(C^{*}\right)^{2}$. Since $S \rightarrow n \mathrm{CP}^{2}$ is an equivariant and generic orbit of the $T^{2}$-action on $n \mathrm{CP}^{2}$ is $T^{2}$ itself, we may assume that $g \notin U(1)^{2}$. Then $\left\{g^{n} \mid n \in Z\right\}$ becomes an infinete cyclic group in $\left(\mathrm{C}^{*}\right)^{2}$. But this is a contradiction because the $\left(\mathrm{C}^{*}\right)^{2}$-action is meromorphic. (Note that the action has a fixed point.) Hence two-dimensional orbit of the $\left(\mathrm{C}^{*}\right)^{2}$-action must be $\left(\mathrm{C}^{*}\right)^{2}$ itself, and this implies $S$ is a toric surface.

\section{Main construction}

In this section we give a construction which is needed to prove that PedersenPoon twistor spaces satisfy Condition (A).

We recall the situation again. Let $n$ be any positive integer and $\alpha_{n}$ be any effective $T^{2}$-action on $n \mathrm{CP}^{2}$. Let $g$ be a self-dual metric on $n \mathrm{CP}^{2}$ and assume that $\alpha_{n}$ is an isometric action with respect to $g$. Let $Z_{1}$ be the twistor space of 
$\left(n \mathrm{CP}^{2}, g\right), \pi_{1}: Z_{1} \rightarrow n \mathrm{CP}^{2}$ the twistor fibration and $\sigma_{1}$ the real structure of $Z_{1} . \alpha_{n}$ naturally induces a holomorphic action on $Z_{1}$ which we also denote by $\alpha_{n}$. We assume that $Z_{1}$ satisfies Condition (A) (see Definition 2.1). Further we suppose that $H^{2}\left(Z_{1}, \Theta_{Z_{1}} \otimes F_{1}^{-1}\right)=0$.

On the other hand let $\alpha_{1}$ be the $T^{2}$-action on $\mathrm{CP}^{2}$ defined in $\S 1.1$, and $Z_{2}:=\mathrm{F}$ the twistor space of $C P^{2}$ (cf. Example 2.4). Let $\pi_{2}, \sigma_{2}$ have similar meanings to $\pi_{1}, \sigma_{1}$.

Any of the fixed points of $\alpha_{n}$ on $n \mathrm{CP}^{2}$ (resp. $\alpha_{1}$ on $\mathrm{CP}^{2}$ ) is isolated and the number of the fixed points is $n+2$ (resp. 3) (cf. §1.1). Let $p_{1} \in n \mathrm{CP}^{2}$ and $p_{2} \in \mathrm{CP}^{2}$ be any of fixed points of $\alpha_{n}$ and $\alpha_{1}$ respectively. Let $\alpha_{n+1}$ be the $T^{2}$-action on $(n+1) \mathrm{CP}^{2}$ obtained as the $T^{2}$-equivariant connected sum of $\left(n \mathrm{CP}^{2}, \alpha_{n}\right)$ and $\left(\mathrm{CP}^{2}, \alpha_{1}\right)$ at $p_{1}$ and $p_{2}$.

Put $L_{i}:=\pi^{-1}\left(p_{i}\right)(i=1,2)$ and let $\mu_{i}: Z_{i}^{\prime} \rightarrow Z_{i}$ be the blowing-ups along $L_{i}$ and $Q_{i}\left(\simeq \mathrm{CP}^{1} \times \mathrm{CP}^{1}\right)$ the exceptional divisors. Let $\rho: Q_{1} \rightarrow Q_{2}$ be a $T^{2}$-equivariant biholomorphic map which preserves the real structures and interchanges the directions of fibers of $\mu_{1}$ and $\mu_{2}$. Identifying $Q_{1}$ and $Q_{2}$ by $\rho$ we get a variety

$$
Z^{\prime}:=Z_{1}^{\prime} \cup_{\rho} Z_{2}^{\prime}
$$

which has a natural structure of compact complex space with only normal crossing singularities along $Q:=Q_{1} \simeq Q_{2}$. Let $\sigma^{\prime}$ be the real structure of $Z^{\prime}$ obtained from $\sigma_{1}$ and $\sigma_{2}$, and $\alpha^{\prime}$ the holomorphic $T^{2}$-action on $Z^{\prime}$ obtained from $\alpha_{n}$ and $\alpha_{1}$. Under these setups, we now construct two families $\mathscr{P}_{1}$ and $\mathscr{P}_{2}$ of Cartier divisors on $Z^{\prime}$.

Let $F_{i}=-\frac{1}{2} K_{Z_{i}}(i=1,2)$ be the line bundles over $Z_{i}$. Condition (A) implies that $\left|F_{i}\right|^{T^{2}}$ is pencil and its general members are a non-singular toric surface. Let $C_{i}(i=1,2)$ be the base curve of $\left|F_{i}\right|^{T^{2}}$ (see Proposition 2.8 (i)). By Proposition 2.8 (iv) and (v), there exist unique elements $D_{i}+\bar{D}_{i} \in\left|F_{i}\right|^{T^{2}}$ for $i=1,2$ which contain $L_{i}$. Let $q_{i}$ and $\bar{q}_{i}(i=1,2)$ be the intersection of $C_{i}$ with $L_{i}$. Let $S_{i} \in\left|F_{i}\right|^{T^{2}}(i=1,2)$ be any member other than $D_{i}+\bar{D}_{i}$. Then $S_{i}$ intersects $L_{i}$ transversally at $q_{i}$ and $\bar{q}_{i}$ because $S_{i} \cdot L_{i}=2$ and $L_{i} \nsubseteq \subseteq S_{i}$. (Note that the meanings of subscripts of these $L_{i}, C_{i}$ and $q_{i}$ are different from those which used in the previous section.)

Let $S_{i}^{\prime}(i=1,2)$ be the proper transforms of $S_{i}$ in $Z_{i}^{\prime}$. Then $\left.\mu_{i}\right|_{S_{i}^{\prime}}: S_{i}^{\prime} \rightarrow S_{i}$ is the blowing-up of $S_{i}$ at $q_{i}$ and $\bar{q}_{i}$. Put $l_{i}:=\mu_{i}^{-1}\left(q_{i}\right)\left(\subseteq Q_{i}\right)$ and $\bar{l}_{i}:=\mu_{i}^{-1}\left(\bar{q}_{i}\right)$ $\left(\subseteq Q_{i}\right)$, the exceptional curves. $l_{i}$ and $\bar{l}_{i}$ are also $T^{2}$-invariant.

On the other hand let $D_{i}^{\prime}$ and $\bar{D}_{i}^{\prime}(i=1,2)$ be the proper transforms of $D_{i}$ and $\bar{D}_{i}$ respectively. Then since $D_{i}$ and $\bar{D}_{i}$ intersect transversally along $L_{i}$ (Proposition 2.5), $D_{i}^{\prime}$ and $\bar{D}_{i}^{\prime}$ are disjoint and they define disjoint two sections $\Gamma_{i}:=D_{i}^{\prime} \cap Q_{i}$ and $\bar{\Gamma}_{i}:=\bar{D}_{i}^{\prime} \cap Q_{i}$ of $\left.\mu_{i}\right|_{Q_{i}}: Q_{i} \rightarrow L_{i}$. It is obvious that $\Gamma_{i}$ 
and $\bar{\Gamma}_{i}$ are also $T^{2}$-invariant. Since $\rho$ is $T^{2}$-equivariant, we may assume that

$$
\rho\left(l_{1}\right)=\Gamma_{2}, \rho\left(\bar{l}_{1}\right)=\bar{\Gamma}_{2}, \rho\left(\Gamma_{1}\right)=l_{2}, \rho\left(\bar{\Gamma}_{1}\right)=\bar{l}_{2} .
$$

These indicate that for each $S_{1} \in\left|F_{1}\right|^{T^{2}}$ other than $D_{1}+\bar{D}_{1}$, the divisor

$$
S^{\prime}:=S_{1}^{\prime} \cup_{\rho}\left(D_{2}^{\prime} \sqcup \bar{D}_{2}^{\prime}\right)=D_{2}^{\prime} \cup S_{1}^{\prime} \cup \bar{D}_{2}^{\prime}
$$

is a $T^{2}$-invariant connected Cartier divisor on $Z^{\prime}$. Interchanging the roles of 1 and 2 , we set

$$
S^{\prime \prime}:=\left(D_{1}^{\prime} \sqcup \bar{D}_{1}^{\prime}\right) \cup_{\rho} S_{2}^{\prime}=D_{1}^{\prime} \cup S_{2}^{\prime} \cup \bar{D}_{1}^{\prime},
$$

which is also $T^{2}$-invariant connected Cartier divisor on $Z^{\prime}$. We note that these are $\sigma^{\prime}$-invariant if we choose $\sigma_{i}$-invariant $S_{i} \in|F|^{T^{2}}$. Next we set

$$
\mathscr{P}_{1}:=\left\{\left.S_{1}^{\prime} \cup\left(D_{2}^{\prime} \amalg \bar{D}_{2}^{\prime}\right)\left|S_{1} \in\right| F_{1}\right|^{T^{2}}, S_{1} \neq D_{1}+\bar{D}_{1}\right\}
$$

and

$$
\mathscr{P}_{2}:=\left\{\left.\left(D_{1}^{\prime} \amalg \bar{D}_{1}^{\prime}\right) \cup S_{2}^{\prime}\left|S_{2} \in\right| F_{2}\right|^{T^{2}}, S_{2} \neq D_{2}+\bar{D}_{2}\right\} .
$$

Any element of $\mathscr{P}_{1}$ and $\mathscr{P}_{2}$ is $T^{2}$-invariant (Cartier) divisor. We also note that any element in $\mathscr{P}_{1}$ is $d$-semi-stable. In fact we have

$$
\begin{aligned}
N_{l_{1} / S_{1}^{\prime}} \otimes N_{\Gamma_{2} / D_{2}^{\prime}} & \simeq\left(\left.N_{Q_{1} / Z_{1}^{\prime}}\right|_{l_{1}}\right) \otimes\left(\left.N_{Q_{2} / Z_{2}^{\prime}}\right|_{\Gamma_{2}}\right) \\
& \left.\simeq\left(N_{Q_{1} / Z_{1}^{\prime}} \otimes N_{Q_{2} / Z_{2}^{\prime}}\right)\right|_{\left.l_{1}=\Gamma_{2}\right)} \\
& \left.\simeq \mathscr{O}_{Q}\right|_{l_{1}\left(=\Gamma_{2}\right)} \simeq \mathscr{O}_{l_{1}\left(=\Gamma_{2}\right)}
\end{aligned}
$$

and $N_{\bar{l}_{1} / S_{1}^{\prime}} \otimes N_{\bar{\Gamma}_{2} / \bar{D}_{2}^{\prime}} \simeq \mathscr{O}_{\bar{l}_{1}\left(=\bar{\Gamma}_{2}\right)}$ as well. In the same way we see that any element of $\mathscr{P}_{2}$ is also $d$-semi-stable.

Remark 3.1. We note that if we choose $D_{1}+\bar{D}_{1}$ for $S_{1}$ and $D_{2}+\bar{D}_{2}$ for $S_{2}$, then these define the same divisor $G:=D_{1}^{\prime}+\bar{D}_{1}^{\prime}+D_{2}^{\prime}+\bar{D}_{2}^{\prime}+2 Q$. That is, the parameter spaces of $\mathscr{P}_{1}$ and $\mathscr{P}_{2}$, which are naturally isomorphic to $\mathrm{C}$, can be compactified by adding the same point and hence $\mathscr{P}_{1} \cup G \cup \mathscr{P}_{2}$ can be regarded as a continuous family.

Technically our key result is the following

Proposition 3.2. Let $S_{1} \in\left|F_{1}\right|^{T^{2}}$ (resp. $S_{2} \in\left|F_{2}\right|^{T^{2}}$ ) be any non-singular member and $S^{\prime}:=S_{1}^{\prime} \cup\left(D_{2}^{\prime} \amalg \bar{D}_{2}^{\prime}\right)\left(\right.$ resp. $\left.S^{\prime \prime}:=\left(D_{1}^{\prime} \amalg \bar{D}_{1}^{\prime}\right) \cup S_{2}^{\prime}\right)$ the corresponding element of $\mathscr{P}_{1}\left(\right.$ resp. $\left.\mathscr{P}_{2}\right)$ as above. Then

(i) $S^{\prime}$ and $S^{\prime \prime}$ are stable (in the sense of Kodaira) under any $T^{2}$-equivariant deformation of $Z^{\prime}$,

(ii) $Z^{\prime}$ can be $T^{2}$-equivariantly deformed into a non-singular threefold $Z_{t}$, 
(iii) when $Z^{\prime}$ will be deformed into a non-singular $Z_{t} T^{2}$-equivariantly, both $S^{\prime}$ and $S^{\prime \prime}$ are deformed into $T^{2}$-invariant non-singular toric surfaces in $Z_{t}$.

Note that in the beginning of this section we have assume that $H^{2}\left(\Theta_{Z_{1}} \otimes\right.$ $F_{1}^{-1}$ ) $=0$. Proposition 3.2 will be proved in the next section. (Of course, the points are (i) and (iii); (ii) has already proved in [16]). Here we use Proposition 3.2 to prove

Proposition 3.3 (= Main Theorem (i)). Let $n$ be any positive integer and $\alpha$ be any effective $T^{2}$-action on $n \mathrm{CP}^{2}$. Let $Z$ be any Pedersen-Poon twistor space associated to $\alpha$. Then $Z$ satisfies Condition ( $A$ ).

Propositions 2.8 and 3.3 imply (i)-(iii) of Main Theorem holds.

Proof of Proposition 3.3. We prove by induction on $n$. The case $n=1$ is easy; in this case, Pedersen-Poon twistor space is nothing but $\mathrm{F}$ in Example 2.4 and we have explicitly checked Condition (A) holds for this space. In addition, we know that $H^{2}\left(\Theta_{\mathrm{F}} \otimes F^{-1}\right)=0$. Let $n$ be any positive integer and fix any effective $T^{2}$-action $\alpha_{n}$ on $n \mathrm{CP}^{2}$. Let $Z_{1}$ be any Pedersen-Poon twistor space associated to $\alpha_{n}$. Suppose that $Z_{1}$ satisfies Condition (A) and further assume that $H^{2}\left(\Theta_{Z_{1}} \otimes F_{1}^{-1}\right)=0$. Take any points $p_{1} \in n \mathrm{CP}^{2}$ and $p_{2} \in \mathrm{CP}^{2}$, which are fixed by $\alpha_{n}$ and $\alpha_{1}$ (see $\S 1.1$ ) respectively. Let $\alpha_{n+1}$ be the effective $T^{2}$ action on $(n+1) \mathrm{CP}^{2}$ obtained by $T^{2}$-equivariant connected sum of $\left(n \mathrm{CP}^{2}, \alpha_{n}\right)$ and $\left(\mathrm{CP}^{2}, \alpha_{1}\right)$ at $p_{1}$ and $p_{2}$. Let $Z_{t}$ be a Pedersen-Poon twistor space over $(n+1) \mathrm{CP}^{2}$ associated to $\alpha_{n+1}$. By definition $Z_{t}$ is obtained as a $T^{2}$-equivariant deformation of $Z^{\prime}=Z_{1}^{\prime} \cup Z_{2}^{\prime}$ as above. Since any Pedersen-Poon twistor space is obtained in this way it suffices to show that $Z_{t}$ satisfies Condition (A) together with $H^{2}\left(\Theta_{Z_{t}} \otimes F_{t}^{-1}\right)=0$, where $F_{t}=-\frac{1}{2} K_{Z_{t}}$ be the line bundle over $Z_{t}$.

First we show that $\left|F_{t}\right|^{T^{2}}$ is one-dimensional as a linear system. Let $S_{1} \in$ $\left|F_{1}\right|^{T^{2}}$ be any $\sigma$-invariant irreducible member, which is always non-singular toric surface as noted in Remark 2.9. Let $S^{\prime}=S_{1}^{\prime} \cup\left(D_{2}^{\prime} \amalg \bar{D}_{2}^{\prime}\right) \in \mathscr{P}_{1}$ be the corresponding element. By (i) of Proposition 3.2, there exists $S_{t}$ in $Z_{t}$ which is a deformation of $S^{\prime}$. By (iii) of Proposition 3.2, $S_{t}$ is a $T^{2}$-invariant nonsingular toric surface. Since $S^{\prime}$ is supposed to be real, $S_{t}$ is also real. On the other hand we have $S_{t} \cdot L_{t}=2$, where $L_{t}$ denotes any twistor line in $Z_{t}$. In fact, if we denote the proper transform of a twistor line which is different from $L_{1}$ by $L^{\prime}\left(\subseteq Z^{\prime}\right)$, then we have $S^{\prime} \cdot L^{\prime}=2$. Since intersection numbers are invariant under deformations, we get $S_{t} \cdot L_{t}=2$, as claimed.

We now know that $S_{t}$ is a real divisor with $S_{t} \cdot L_{t}=2$. This implies that $S_{t}$ is a member of $\left|F_{t}\right|^{T^{2}}$. We note that the same argument shows that any real irreducible member $S_{2} \in\left|F_{2}\right|^{T^{2}}$ also gives a real non-singular member 
of $\left|F_{t}\right|^{T^{2}}$. Then as showed in the proof of Proposition 2.8 we have an exact sequence

$$
0 \longrightarrow H^{0}\left(Z_{t}, \mathscr{O}_{Z_{t}}\right)^{T^{2}} \longrightarrow H^{0}\left(Z_{t}, F_{t}\right)^{T^{2}} \longrightarrow H^{0}\left(S_{t},-K_{S_{t}}\right)^{T^{2}} \longrightarrow 0 .
$$

As a consequence, we have $\operatorname{dim} H^{0}\left(Z_{t}, F_{t}\right)^{T^{2}}=2$, as claimed.

Next we show that general members of the pencil $\left|F_{t}\right|^{T^{2}}$ are non-singular toric surface. Proposition 3.2 (i) implies that $\mathscr{P}_{1}$ and $\mathscr{P}_{2}$ on $Z^{\prime}$ induce a onedimensional family $\mathscr{P}$ of $T^{2}$-invariant divisors on $Z_{t}$. By (iii), general element of $\mathscr{P}$ is a non-singular toric surface. As we have shown above at least one element of this family is a member of $\left|F_{t}\right|^{T^{2}}$. On the other hand we have Pic $Z_{t} \subseteq H^{2}\left(Z_{t}, Z\right)$. Consequently this family is contained in $\left|F_{t}\right|^{T^{2}}$, which implies that generic member of $\left|F_{t}\right|^{T^{2}}$ is an element of $\mathscr{P}$.

Finally to work induction we have to show that $H^{2}\left(\Theta_{Z_{t}} \otimes F_{t}^{-1}\right)=0$, since Proposition 3.2 requires the assumption $H^{2}\left(\Theta_{Z_{1}} \otimes F_{1}^{-1}\right)=0$. By [7, Proposition 4.1] we have an isomorphism $H^{2}\left(\Theta_{Z^{\prime}}^{0}\left(-S^{\prime}\right)\right) \simeq H^{2}\left(\Theta_{Z_{1}}\left(-S_{1}\right)\right)$ and the latter is zero by the assumption. Hence we have $H^{2}\left(\Theta_{Z^{\prime}}^{0}\left(-S^{\prime}\right)\right)=0$. Then upper semi-continuity of cohomology groups shows that $H^{2}\left(\Theta_{Z_{t}}^{0}\left(-S_{t}\right)\right)=0$, at least if we shrink a parameter space of deformations sufficiently small.

Remark 3.4. If we choose a reducible element for $S_{i} \in\left|F_{i}\right|^{T^{2}}(i=1$ or 2) other than $D_{i}+\bar{D}_{i}$ then the corresponding $S^{\prime} \in \mathscr{P}_{1}$ and $S^{\prime \prime} \in \mathscr{P}_{2}$ consist of four irreducible components. The number of such elements in $\mathscr{P}_{1}$ (resp. $\mathscr{P}_{2}$ ) is $(n+2)-1=n+1$ (resp. $3-1=2$ ). All the other elements in $\mathscr{P}_{1} \cup \mathscr{P}_{2}$ has three irreducible components. Hence there exist $(n+1)+2=n+3$ elements in $\mathscr{P}_{1} \cup \mathscr{P}_{2}$ which are "special" in the sense that the number of irreducible components is four (Proposition 3.!!), and this number coincides with the number of the reducible members of $\left|F_{t}\right|^{T^{2}}$. It is conceivable, though we do not prove, that "special" elements of $\mathscr{P}_{1} \cup \mathscr{P}_{2}$ exactly give reducible members in $\left|F_{t}\right|^{T^{2}}$. (See also Remark 3.1.)

\section{Proof of Proposition 3.2}

In this section we prove Proposition 3.2. As in Proposition 3.3, Proposition 3.2 implies that (i)-(iii) of Main Theorem hold true. We continue to use the notations used in the previous section.

Let $Z^{\prime}=Z_{1}^{\prime} \cup Z_{2}^{\prime}$ be the compact complex space (with only simple normal crossing singularities along $Q$ ) which was constructed from $Z_{1} \rightarrow n \mathrm{CP}^{2}$ (with $\alpha_{n}$ ) and $Z_{2}=\mathrm{F} \rightarrow \mathrm{CP}^{2}$ (with $\left.\alpha_{1}\right)$. $Z^{\prime}$ has the holomorphic $T^{2}$-action $\alpha^{\prime}$ and there exist two families $\mathscr{P}_{1}$ and $\mathscr{P}_{2}$ whose elements are $T^{2}$-invariant Cartier divisors on $Z^{\prime} . \mathscr{P}_{1}$ and $\mathscr{P}_{2}$ have constructed from the pencils $\left|F_{1}\right|^{T^{2}}$ and $\left|F_{2}\right|^{T^{2}}$ respectively. Let $S_{1} \in\left|F_{1}\right|^{T^{2}}$ be any non-singular member and 
$S^{\prime}:=S_{1}^{\prime} \cup\left(D_{2}^{\prime} \sqcup \bar{D}_{2}^{\prime}\right)$ be the corresponding element of $\mathscr{P}_{1}$. Similarly, let $S^{\prime \prime}:=\left(D_{1}^{\prime} \amalg \bar{D}_{1}^{\prime}\right) \cup S_{2}^{\prime}$ be the element of $\mathscr{P}_{2}$ for non-singular $S_{2} \in\left|F_{2}\right|^{T^{2}}$. Further, we have supposed that $H^{2}\left(\Theta_{Z_{1}} \otimes F_{1}^{-1}\right)=0$, from which it follows that

$$
H^{2}\left(Z^{\prime}, \Theta_{Z^{\prime}}^{0}\left(-S^{\prime}\right)\right)=0
$$

as seen in the last paragraph of the proof of Proposition 3.3.

The first half of this section is devoted to prove the following

Proposition 4.1 (= Proposition 3.2 (i)). $S^{\prime}$ and $S^{\prime \prime}$ are stable under any $T^{2}$-equivariant deformation of $Z^{\prime}$.

Although we prove the above result only for $S^{\prime} \in \mathscr{P}_{1}$, the same proof works for $S^{\prime \prime} \in \mathscr{P}_{2}$ by interchanging the roles of 1 and 2 .

To prove this, we need to study

- ( $T^{2}$-equivariant) deformations of $Z^{\prime}$,

- $\left(T^{2}\right.$-equivariant $)$ deformations of $S^{\prime}$,

- ( $T^{2}$-equivariant) deformations of the pair $\left(Z^{\prime}, S^{\prime}\right)$, and

- relation between these three deformations.

The following three sheaves are fundamental for these purposes:

- $\Omega_{Z^{\prime}}\left(\right.$ resp. $\Omega_{S^{\prime}}$ ): the sheaf of Kähler differentials of $Z^{\prime}$ (resp. $S^{\prime}$ ). If we embed $Z^{\prime}$ into $C^{4}\left(\right.$ resp. $\left.C^{3}\right)$ as a hypersurface $\left\{\left(z_{1}, z_{2}, z_{3}, z_{4}\right) \in C^{4} \mid z_{1} z_{2}=0\right\}$ (resp. $\left.\left\{\left(z_{1}, z_{2}, z_{3}\right) \in C^{3} \mid z_{1} z_{2}=0\right\}\right)$, then $\Omega_{Z^{\prime}}\left(\right.$ resp. $\left.\Omega_{S^{\prime}}\right)$ is defined as the cokernel of the natural injection $0 \rightarrow \mathscr{I}_{Z^{\prime}} /\left.\mathscr{I}_{Z^{\prime}}^{2} \stackrel{d}{\rightarrow} \Omega_{C^{4}}\right|_{Z^{\prime}}$ (resp. $0 \rightarrow$ $\mathscr{I}_{S^{\prime}} / \mathscr{I}_{S^{\prime}}^{2} \stackrel{d}{\rightarrow} \Omega_{C^{3}} \mid S_{S^{\prime}}$ ), where $\mathscr{I}_{Z^{\prime}}$ (resp. $\mathscr{I}_{S^{\prime}}$ ) denotes the ideal sheaf of $Z^{\prime}$ (resp. $\left.S^{\prime}\right)$ in $C^{4}$ (resp. $C^{3}$ ).

- $\Omega_{Z^{\prime}, S^{\prime}}=\Omega_{Z^{\prime}}\left(\log S^{\prime}\right)$ : the sheaf of Kähler differentials of $Z^{\prime}$ which have at worst logarithmic poles along $S^{\prime}$. If $S^{\prime}$ is defined in $C^{4}$ as a complete intersection $S^{\prime}=Z^{\prime} \cap H$, where $H$ is a hypersurface defined by $\left\{z_{4}=0\right\}$, then $\Omega_{Z^{\prime}, S^{\prime}}$ is defined as the cokernel of a natural injection $0 \rightarrow \mathscr{I}_{Z^{\prime}} /\left.\mathscr{I}_{Z^{\prime}}^{2} \stackrel{d}{\rightarrow} \Omega_{C^{4}, H}\right|_{Z^{\prime}}$, where $\Omega_{C^{4}, H}$ denotes the sheaf of Kähler differentials of $C^{4}$ which have at worst logarithmic poles along $H$ (that is, $\Omega_{\mathrm{C}^{4}, H}$ is the free $\mathscr{O}_{\mathrm{C}^{4}}$-module generated by $\left.\left\{d z_{1}, d z_{2}, d z_{3}, d z_{4} / z_{4}\right\}\right)$. 
These three sheaves fit into the following exact and commutative diagrams:

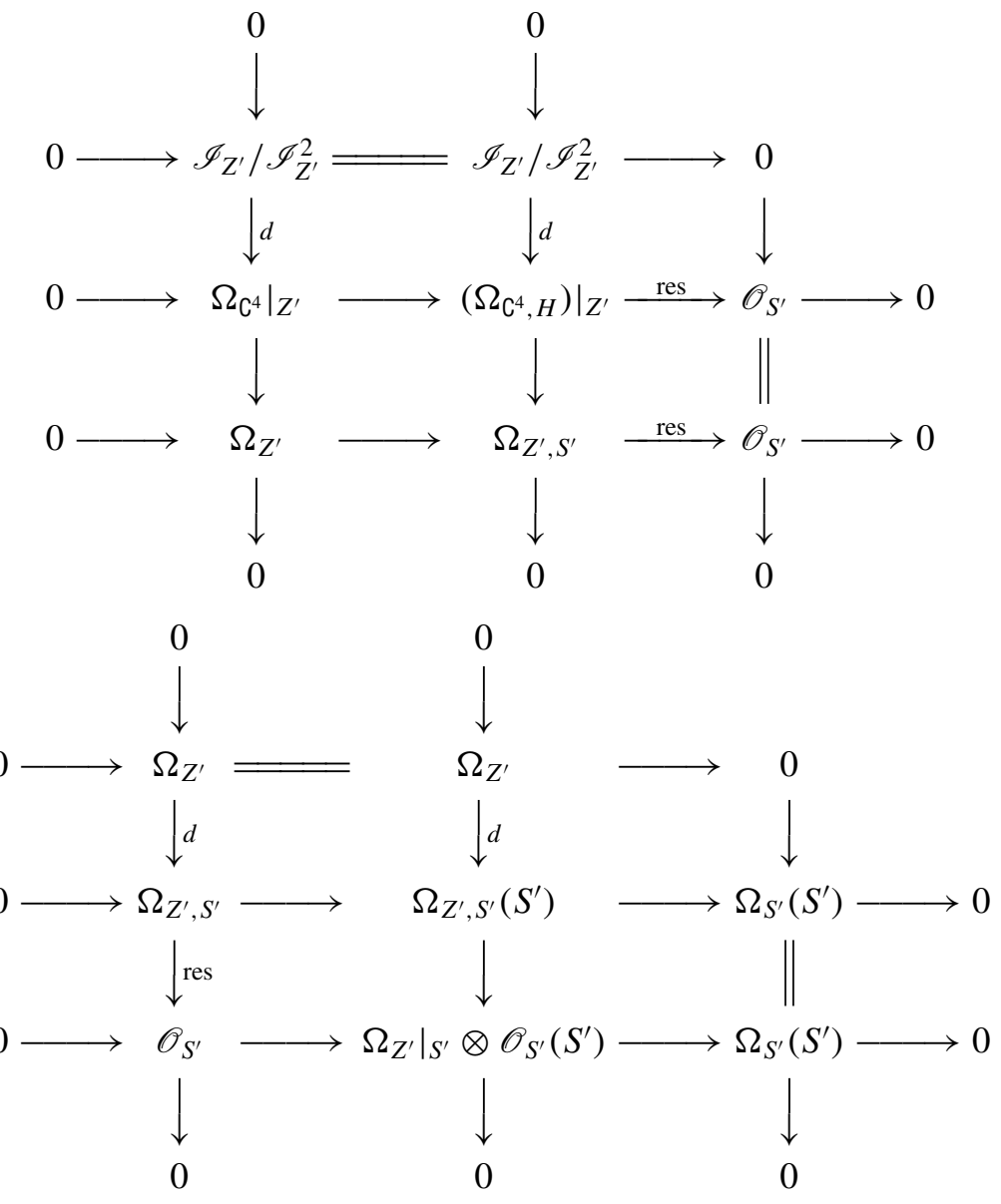

(If $S^{\prime}$ were a non-singular hypersurface in a non-singular $Z^{\prime}$, then the last row of the first diagram would be called the Poincaré residue sequence.)

In addition, we use the following notations:

$$
\begin{aligned}
& \Theta_{Z^{\prime}}^{i}:=\mathscr{E} x t_{\mathscr{O}_{Z^{\prime}}}^{i}\left(\Omega_{Z^{\prime}}, \mathscr{O}_{Z^{\prime}}\right), \Theta_{S^{\prime}}^{i}:=\mathscr{E} x t_{\mathscr{O}_{S^{\prime}}}^{i}\left(\Omega_{S^{\prime}}, \mathscr{O}_{S^{\prime}}\right), \\
& \Theta_{Z^{\prime}, S^{\prime}}^{i}:=\mathscr{E} x t_{\mathscr{O}_{Z^{\prime}}}^{i}\left(\Omega_{Z^{\prime}, S^{\prime}}, \mathscr{O}_{Z^{\prime}}\right), \\
& T_{Z^{\prime}}^{i}:=\operatorname{Ext}_{Z^{\prime}}^{i}\left(\Omega_{Z^{\prime}}, \mathscr{O}_{Z^{\prime}}\right), T_{S^{\prime}}^{i}:=\operatorname{Ext}_{S^{\prime}}^{i}\left(\Omega_{S^{\prime}}, \mathscr{O}_{S^{\prime}}\right), \\
& T_{Z^{\prime}, S^{\prime}}^{i}:=\operatorname{Ext}_{Z^{\prime}}^{i}\left(\Omega_{Z^{\prime}, S^{\prime}}, \mathscr{O}_{Z^{\prime}}\right) .
\end{aligned}
$$

Deformation theories of compact complex spaces imply that $T_{Z^{\prime}}^{1}$ (resp. $T_{Z^{\prime}}^{1}$ and resp. $T_{Z^{\prime}, S^{\prime}}^{1}$ ) is the space of first order deformations of $Z^{\prime}$ (resp. $S^{\prime}$ and resp. the pair $\left.\left(Z^{\prime}, S^{\prime}\right)\right)$ and that $T_{Z^{\prime}}^{2}\left(\operatorname{resp} . T_{S^{\prime}}^{2}\right.$ and resp. $\left.T_{Z^{\prime}, S^{\prime}}^{2}\right)$ is the obstruction space. In particular, if $T_{Z^{\prime}}^{2}=0\left(\operatorname{resp} . T_{S^{\prime}}^{2}=0\right.$ and resp. $\left.T_{Z^{\prime}, S^{\prime}}^{2}=0\right)$, then any 
first order deformation extends to an actual deformation of $Z^{\prime}$ (resp. the pair $S^{\prime}$ and resp. the pair $\left(Z^{\prime}, S^{\prime}\right)$ ). When a compact Lie group $G$ acts on $Z^{\prime}$ (resp. $\left(Z^{\prime}, S^{\prime}\right)$; that is $S^{\prime}$ is a $G$-invariant subspace of $\left.Z^{\prime}\right)$, equivariant deformation theory [2] implies that if $T_{Z^{\prime}}^{2}=0$ (resp. $T_{S^{\prime}}^{2}=0$ and resp. $T_{Z^{\prime}, S^{\prime}}^{2}=0$ ), then $\left(T_{Z^{\prime}}^{1}\right)^{G}$ (resp. $\left(T_{S^{\prime}}^{1}\right)^{G}$ and resp. $\left(T_{Z^{\prime}, S^{\prime}}^{1}\right)^{G}$ ) is the space of Kuranishi family of $G$-equivariant deformations of $Z^{\prime}$ (resp. the pair $S^{\prime}$ and resp. the pair $\left(Z^{\prime}, S^{\prime}\right)$ ).

To calculate these spaces, we use the local to global spectral sequences:

$$
\begin{aligned}
E_{2}^{p, q} & :=H^{p}\left(Z^{\prime}, \Theta_{Z^{\prime}}^{q}\right) \Rightarrow T_{Z^{\prime}}^{p+q}, \\
E_{2}^{p, q} & :=H^{p}\left(S^{\prime}, \Theta_{S^{\prime}}^{q}\right) \Rightarrow T_{S^{\prime}}^{p+q}
\end{aligned}
$$

and

$$
E_{2}^{p, q}:=H^{p}\left(Z^{\prime}, \Theta_{Z^{\prime}, S^{\prime}}^{q}\right) \Rightarrow T_{Z^{\prime}, S^{\prime}}^{p+q} .
$$

Before studying deformations of $Z^{\prime}$, we study deformations of $S^{\prime}$.

Lemma 4.2. We have $H^{2}\left(\Theta_{S^{\prime}}^{0}\right)=T_{S^{\prime}}^{2}=0$ and there exists a $T^{2}$-equivariant exact sequence

$$
0 \longrightarrow H^{1}\left(\Theta_{S^{\prime}}^{0}\right) \longrightarrow T_{S^{\prime}}^{1} \longrightarrow H^{0}\left(\Theta_{S^{\prime}}^{1}\right) \longrightarrow 0 .
$$

Proof. Since $S_{1}^{\prime}$ and $D_{2}^{\prime}$ are rational surfaces, we have $H^{2}\left(\Theta_{S_{1}^{\prime}}\right)=H^{2}\left(\Theta_{D_{2}^{\prime}}\right)$ $=0$. Because the degree of $l_{1}$ and $\Gamma_{2}$ are $(-1)$ and $(+1)$-curves of $S_{1}^{\prime}$ and $D_{2}^{\prime}$ respectively, we have $H^{i}\left(N_{l_{1} / S_{1}^{\prime}}\right)=H^{i}\left(N_{\Gamma_{2} / D_{2}^{\prime}}\right)=0$ for any $i \geq 1$. Hence the cohomology exact sequences of the following two exact sequences:

$$
\begin{gathered}
0 \longrightarrow \Theta_{S_{1}^{\prime}, l_{1}+\bar{l}_{1}} \longrightarrow \Theta_{S_{1}^{\prime}} \longrightarrow N_{l_{1} / S_{1}^{\prime}} \oplus N_{\bar{l}_{1} / S_{1}^{\prime}} \longrightarrow 0, \\
0 \longrightarrow \Theta_{D_{2}^{\prime}, \Gamma_{2}} \longrightarrow \Theta_{D_{2}^{\prime}} \longrightarrow N_{\Gamma_{2} / D_{2}^{\prime}} \longrightarrow 0
\end{gathered}
$$

together with the reality imply that $H^{2}\left(\Theta_{S_{1}^{\prime}, l_{1}+\bar{l}_{1}}\right)=H^{2}\left(\Theta_{D_{2}^{\prime}, \Gamma_{2}}\right)=H^{2}\left(\Theta_{\bar{D}_{2}^{\prime}, \bar{\Gamma}_{2}}\right)$ $=0$. Consequently the cohomology exact sequence of

$$
0 \rightarrow \Theta_{S^{\prime}}^{0} \rightarrow v_{*}\left(\Theta_{S_{1}^{\prime}, l_{1}+\bar{l}_{1}} \oplus \Theta_{D_{2}^{\prime}, \Gamma_{2}} \oplus \Theta_{\bar{D}_{2}^{\prime}, \bar{\Gamma}_{2}}\right) \longrightarrow \Theta_{l} \oplus \Theta_{\bar{l}} \longrightarrow 0
$$

and the Leray spectral sequence show that $H^{2}\left(\Theta_{S^{\prime}}^{0}\right)=0$, where we denote $l:=l_{1} \simeq \Gamma_{2}$ and $\bar{l}:=\bar{l}_{1} \simeq \bar{\Gamma}_{2}$. Next it is easily verified that

$$
\Theta_{S^{\prime}}^{i}:=\mathscr{E} x t_{\mathscr{O}_{S^{\prime}}}^{i}\left(\Omega_{S^{\prime}}, \mathscr{O}_{S^{\prime}}\right) \simeq \begin{cases}\mathscr{O}_{l} \oplus \mathscr{O}_{\bar{l}}, & i=1, \\ 0, & i \geq 2 .\end{cases}
$$


Hence the local to global spectral sequence associated to the hypercohomology groups $T_{S^{\prime}}^{i}:=\operatorname{Ext}_{S^{\prime}}^{i}\left(\Omega_{S^{\prime}}, \mathscr{O}_{S^{\prime}}\right)$ induces the long exact sequence

$0 \longrightarrow H^{1}\left(\Theta_{S^{\prime}}^{0}\right) \longrightarrow T_{S^{\prime}}^{1} \longrightarrow H^{0}\left(\Theta_{S^{\prime}}^{1}\right) \longrightarrow H^{2}\left(\Theta_{S^{\prime}}^{0}\right) \longrightarrow T_{S^{\prime}}^{2} \longrightarrow H^{1}\left(\Theta_{S^{\prime}}^{1}\right)$.

From this, the claims of the lemma follow.

Next we prove

Lemma 4.3. $H^{1}\left(\Theta_{S^{\prime}}^{0}\right)^{T^{2}}=0$ and $\left(T_{S^{\prime}}^{1}\right)^{T^{2}} \simeq H^{0}\left(\Theta_{S^{\prime}}^{1}\right) \simeq \mathrm{C}^{2}$.

PROOF. Since $D_{2}^{\prime} \simeq D_{2}$ is a non-singular toric surface and $\Gamma_{2}$ is one of its onedimensional orbit of the $\left(C^{*}\right)^{2}$-action, we see readily that $\operatorname{dim} H^{0}\left(\Theta_{D_{2}^{\prime}, \Gamma_{2}}\right)^{T^{2}}$ $=2$ and $\operatorname{dim} H^{0}\left(\Theta_{\Gamma_{2}}\right)^{T^{2}}=1$ and that the restriction map $H^{0}\left(\Theta_{D_{2}^{\prime}, \Gamma_{2}}\right)^{T^{2}} \rightarrow$ $H^{0}\left(\Theta_{\Gamma_{2}}\right)^{T^{2}}$ is surjective. Hence the cohomology exact sequence of (12) implies

$$
H^{1}\left(\Theta_{S^{\prime}}^{0}\right)^{T^{2}} \simeq H^{1}\left(\Theta_{S_{1}^{\prime}, l_{1}+\bar{l}_{1}}\right)^{T^{2}} \oplus H^{1}\left(\Theta_{D_{2}^{\prime}, \Gamma_{2}}\right)^{T^{2}} \oplus H^{1}\left(\Theta_{\bar{D}_{2}^{\prime}, \bar{\Gamma}_{2}}\right)^{T^{2}} .
$$

We now show that all the terms of the right-hand-side vanish. The cohomology exact sequence of (10) induces a $T^{2}$-equivariant isomorphism $H^{1}\left(\Theta_{S_{1}^{\prime}, l_{1}+\bar{l}_{1}}\right) \simeq$ $H^{1}\left(\Theta_{S_{1}^{\prime}}\right)$. But since $S_{1}^{\prime}$ is a non-singular toric surface, we have $H^{1}\left(\Theta_{S_{1}^{\prime}}\right)^{T^{2}}=0$. Hence we have $H^{1}\left(\Theta_{S_{1}^{\prime}, l_{1}+\bar{l}_{1}}\right)^{T^{2}}=0$. Next, because $D_{2}^{\prime}$ is biholomorphic to the non-minimal Hirzebruch surface, we can easily show that the map $H^{0}\left(\Theta_{D_{2}^{\prime}}\right) \rightarrow$ $H^{0}\left(N_{\Gamma_{2} / D_{2}^{\prime}}\right)$ is surjective. Hence by (11) we have $T^{2}$-equivariant isomorphisms $H^{1}\left(\Theta_{D_{2}^{\prime}, \Gamma_{2}}\right) \simeq H^{1}\left(\Theta_{D_{2}^{\prime}}\right)$ and by reality $H^{1}\left(\Theta_{\bar{D}_{2}^{\prime}, \bar{\Gamma}_{2}}\right) \simeq H^{1}\left(\Theta_{\bar{D}_{2}^{\prime}}\right)$. But as in $S_{1}^{\prime}$, we have $H^{1}\left(\Theta_{D_{2}^{\prime}}\right)^{T^{2}}=H^{1}\left(\Theta_{\bar{D}_{2}^{\prime}}\right)^{T^{2}}=0$. Therefore we have $H^{1}\left(\Theta_{S^{\prime}}^{0}\right)^{T^{2}}=0$.

For the latter claim, we note that since $\Theta_{S^{\prime}}^{1}$ is naturally identified with $\left.\Theta_{Z^{\prime}}^{1}\right|_{S^{\prime}}\left(\simeq \mathscr{O}_{l} \oplus \mathscr{O}_{\bar{l}}\right)$ and the $T^{2}$-action on $H^{0}\left(\Theta_{Z^{\prime}}^{1}\right) \simeq \mathrm{C}$ is trivial, we have $H^{0}\left(\Theta_{S^{\prime}}^{1}\right)^{T^{2}} \simeq H^{0}\left(\Theta_{S^{\prime}}^{1}\right) \simeq C^{2}$. Then the first claim and the exact sequence of Lemma 4.2 give rise to the required isomorphism $\left(T_{S^{\prime}}^{1}\right)^{T^{2}} \simeq H^{0}\left(\Theta_{S^{\prime}}^{1}\right) \simeq C^{2}$.

Let $p: \mathscr{S} \rightarrow B$ with $p^{-1}(0) \simeq S^{\prime}$ be the Kuranishi family of $T^{2}$ equivariant deformations of $S^{\prime}$. Lemmas 4.2 and 4.3 show that $B$ can be regarded as a sufficiently small open disk in $\left(T_{S^{\prime}}^{1}\right)^{T^{2}} \simeq \mathrm{C}^{2}$. Corresponding to the $T^{2}$-equivariant decomposition $\left(T_{S^{\prime}}^{1}\right)^{T^{2}}\left(\simeq H^{0}\left(\Theta_{S^{\prime}}^{1}\right) \simeq\left(H^{0}\left(\mathscr{O}_{l} \oplus \mathscr{O}_{\bar{l}}\right)\right) \simeq \mathrm{C} \oplus \mathrm{C}\right.$, there exist two non-singular holomorphic curves $B_{1}$ and $B_{2}$ in $B$ which intersect transversally at 0 .

Proposition 4.4. Let $p: \mathscr{S} \rightarrow B \supseteq B_{1}, B_{2}$ be as above. If $t \notin B_{1} \cup B_{2}$ then $p^{-1}(t)$ is a non-singular toric surface which is biholomorphic to $S_{1}^{\prime}$.

Proof. Assume $t \notin B_{1} \cup B_{2}$. Then $S_{t}:=p^{-1}(t)$ is non-singular by a result of [4]. By upper semi-continuity of cohomology groups and the classification 
of compact complex surfaces, we can easily show that $S_{t}$ is a rational surface. Then an argument used in Remark 2.9 shows that $S_{t}$ is a toric surface. On the other hand, as in the argument in the proof of [7, Proposition 1.3], $S^{\prime}$ admits a deformation $p_{0}: \mathscr{S}_{0} \rightarrow \mathrm{C}$ such that $p_{0}^{-1}(t) \simeq S_{1}^{\prime}$ for every $t \neq 0$. Since every $T^{2}$-equivariant deformation of toric surface is the trivial deformation (which follows from the rationality of the cones of the fan $\Delta \subseteq N_{\mathrm{R}}$ (cf. §1.2)), $S_{t}$ must be biholomorphic to $S_{1}^{\prime}$.

Next we recall deformation theory of $Z^{\prime}$.

Lemma 4.5 ([3], [4]).

$$
\Theta_{Z^{\prime}}^{i} \simeq \begin{cases}\mathscr{O}_{Q}, & i=1 \\ 0, & i \geq 2\end{cases}
$$

Lemma $4.6([3],[4]) . H^{2}\left(\Theta_{Z^{\prime}}^{0}\right)=T_{Z^{\prime}}^{2}=0$ and we have a $T^{2}$-equivariant exact sequence

$$
0 \longrightarrow H^{1}\left(\Theta_{Z^{\prime}}^{0}\right) \longrightarrow T_{Z^{\prime}}^{1} \longrightarrow H^{0}\left(\Theta_{Z^{\prime}}^{1}\right) \longrightarrow 0 .
$$

Lemma 4.7 ([16]). The natural $T^{2}$-action on $H^{0}\left(\Theta_{Z^{\prime}}^{1}\right) \simeq \mathrm{C}$ is the trivial action.

Next we study ( $T^{2}$-equivariant) deformations of the pair $\left(Z^{\prime}, S^{\prime}\right)$.

The following result was used in [7] and [8].

LeMma 4.8. For any $i \geq 1$ we have a canonical $T^{2}$-equivariant isomorphism

$$
\Theta_{Z^{\prime}}^{i} \simeq \Theta_{Z^{\prime}, S^{\prime}}^{i}
$$

Proof. Since $\mathscr{O}_{Z^{\prime}}$ and $\mathscr{O}_{Z^{\prime}}\left(-S^{\prime}\right)$ are locally free $\mathscr{O}_{Z^{\prime}}$-modules, we have $T^{2}$-equivariant isomorphisms

$$
\mathscr{E} x t_{\mathscr{O}_{Z^{\prime}}}^{i}\left(\mathscr{O}_{Z^{\prime}}, \mathscr{O}_{Z^{\prime}}\right) \simeq \begin{cases}\mathscr{O}_{Z^{\prime}} & i=0 \\ 0 & i \geq 1\end{cases}
$$

and

$$
\mathscr{E} x t_{\mathscr{O}_{Z^{\prime}}}^{i}\left(\mathscr{O}_{Z^{\prime}}\left(-S^{\prime}\right), \mathscr{O}_{Z^{\prime}}\right) \simeq \begin{cases}\mathscr{O}_{Z^{\prime}}\left(S^{\prime}\right) & i=0 \\ 0 & i \geq 1\end{cases}
$$

On the other hand we have

$$
\mathscr{E} x t_{\mathscr{O}_{Z^{\prime}}}^{0}\left(\mathscr{O}_{S^{\prime}}, \mathscr{O}_{Z^{\prime}}\right) \simeq \mathscr{H} o m_{\mathscr{O}_{Z^{\prime}}}\left(\mathscr{O}_{S^{\prime}}, \mathscr{O}_{Z^{\prime}}\right)=0 .
$$


Indeed, since $S^{\prime}$ is a Cartier divisor, we may choose a local defining equation $f \in \mathscr{O}_{Z^{\prime}}$ of $S^{\prime}$. Let $\varphi: \mathscr{O}_{S^{\prime}} \rightarrow \mathscr{O}_{Z^{\prime}}$ be any homomorphism over $\mathscr{O}_{Z^{\prime}}$ and $g \in \mathscr{O}_{S^{\prime}}$ be any element. Then we have $f \cdot \varphi(g)=\varphi(f g)=\varphi(0)=0$. This implies $\varphi(g)=0$. Next we consider the obvious $T^{2}$-equivariant exact sequence

$$
0 \longrightarrow \mathscr{O}_{Z^{\prime}}\left(-S^{\prime}\right) \longrightarrow \mathscr{O}_{Z^{\prime}} \longrightarrow \mathscr{O}_{S^{\prime}} \longrightarrow 0 .
$$

Applying the functor $\mathscr{H} \mathrm{m}_{\mathscr{O}_{Z^{\prime}}}\left(\cdot, \mathscr{O}_{Z^{\prime}}\right)$ to this and using (13), (14) and (15), we get a $T^{2}$-equivariant exact sequence

$$
0 \longrightarrow \mathscr{O}_{Z^{\prime}} \longrightarrow \mathscr{O}_{Z^{\prime}}\left(S^{\prime}\right) \longrightarrow \mathscr{E} x t_{\mathscr{Z}_{Z^{\prime}}}^{1}\left(\mathscr{O}_{S^{\prime}}, \mathscr{O}_{Z^{\prime}}\right) \longrightarrow 0
$$

and for any $i \geq 2$

$$
\mathscr{E} x t_{\mathscr{O}_{Z^{\prime}}}^{i}\left(\mathscr{O}_{S^{\prime}}, \mathscr{O}_{Z^{\prime}}\right)=0
$$

Moreover, (16) shows that

$$
\mathscr{E} x t_{\mathscr{O}_{Z^{\prime}}}^{1}\left(\mathscr{O}_{S^{\prime}}, \mathscr{O}_{Z^{\prime}}\right) \simeq \mathscr{O}_{S^{\prime}}\left(S^{\prime}\right)
$$

Next we consider the "Poincaré residue sequence"

$$
0 \longrightarrow \Omega_{Z^{\prime}} \longrightarrow \Omega_{Z^{\prime}, S^{\prime}} \stackrel{\text { res }}{\longrightarrow} \mathscr{O}_{S^{\prime}} \longrightarrow 0,
$$

which is also $T^{2}$-equivariant. Applying the functor $\mathscr{H}_{o m_{Z_{Z^{\prime}}}}\left(\cdot, \mathscr{O}_{Z^{\prime}}\right)$ to this and using (15), (18) and (17), we obtain a $T^{2}$-equivariant exact sequence

$$
0 \longrightarrow \Theta_{Z^{\prime}, S^{\prime}}^{0} \longrightarrow \Theta_{Z^{\prime}}^{0} \stackrel{\alpha}{\longrightarrow} \mathscr{O}_{S^{\prime}}\left(S^{\prime}\right) \longrightarrow \Theta_{Z^{\prime}, S^{\prime}}^{1} \longrightarrow \Theta_{Z^{\prime}}^{1} \longrightarrow 0
$$

and $T^{2}$-equivariant isomorphisms $\Theta_{Z^{\prime}, S^{\prime}}^{i} \simeq \Theta_{Z^{\prime}}^{i}$ for any $i \geq 2$. Hence to complete a proof, it suffices to show that $\alpha$ is surjective. To see this, we choose a complex coordinate $\left(z_{1}, z_{2}, z_{3}, z_{4}\right)$ on $\mathrm{C}^{4}$ such that

$$
Z^{\prime}=\left\{z_{1} z_{2}=0\right\} \quad \text { and } \quad S^{\prime}=\left\{z_{1} z_{2}=z_{4}=0\right\}
$$

as before. Then $\Theta_{Z^{\prime}}^{0}$ is generated by $\left\{z_{1} \partial_{1}, z_{2} \partial_{2}, \partial_{3}\right.$, and $\left.\partial_{4}\right\}$, and $\Theta_{Z^{\prime}, S^{\prime}}^{0}$ is generated by $\left\{z_{1} \partial_{1}, z_{2} \partial_{2}, \partial_{3}\right.$, and $\left.z_{4} \partial_{4}\right\}$, where we set $\partial_{i}:=\partial / \partial z_{i}$. These imply that the cokernel of the natural injection $\Theta_{Z^{\prime}, S^{\prime}}^{0} \rightarrow \Theta_{Z^{\prime}}^{0}$ is $\mathscr{O}_{S^{\prime}} \partial / \partial z_{4}$, which is naturally isomorphic to $\mathscr{O}_{S^{\prime}}\left(S^{\prime}\right)$. Therefore $\alpha$ is surjective and we get from (20) the required isomorphism $\Theta_{Z^{\prime}, S^{\prime}}^{1} \simeq \Theta_{Z^{\prime}}^{1}$.

Lemma 4.9. $H^{2}\left(\Theta_{Z^{\prime}, S^{\prime}}^{0}\right)=T_{Z^{\prime}, S^{\prime}}^{2}=0$ and we have a $T^{2}$-equivariant exact sequence

$$
0 \longrightarrow H^{1}\left(\Theta_{Z^{\prime}, S^{\prime}}^{0}\right) \longrightarrow T_{Z^{\prime}, S^{\prime}}^{1} \longrightarrow H^{0}\left(\Theta_{Z^{\prime}, S^{\prime}}^{1}\right) \longrightarrow 0 .
$$


Proof. Lemmas 4.8 and 4.5 imply $\Theta_{Z^{\prime}, S^{\prime}}^{i}=0$ for any $i \geq 2$. Therefore local to global spectral sequence yields a $T^{2}$-equivariant exact sequence

$$
\begin{aligned}
0 \rightarrow H^{1}\left(\Theta_{Z^{\prime}, S^{\prime}}^{0}\right) \rightarrow T_{Z^{\prime}, S^{\prime}}^{1} \rightarrow & H^{0}\left(\Theta_{Z^{\prime}, S^{\prime}}^{1}\right) \\
& \rightarrow H^{2}\left(\Theta_{Z^{\prime}, S^{\prime}}^{0}\right) \rightarrow T_{Z^{\prime}, S^{\prime}}^{2} \rightarrow H^{1}\left(\Theta_{Z^{\prime}, S^{\prime}}^{1}\right) .
\end{aligned}
$$

Now we have $H^{2}\left(\Theta_{Z^{\prime}}^{0}\left(-S^{\prime}\right)\right)=0$ (see (9)) and by Lemma $4.2 H^{2}\left(\Theta_{S^{\prime}}^{0}\right)=0$. Then the exact sequence

$$
0 \longrightarrow \Theta_{Z^{\prime}}^{0}\left(-S^{\prime}\right) \longrightarrow \Theta_{Z^{\prime}, S^{\prime}}^{0} \longrightarrow \Theta_{S^{\prime}}^{0} \longrightarrow 0
$$

shows that $H^{2}\left(\Theta_{Z^{\prime}, S^{\prime}}^{0}\right)=0$ and the above long exact sequence implies the required sequence.

Having proved the unobstructedness of deformations of $Z^{\prime}$ and the pair $\left(Z^{\prime}, S^{\prime}\right)$, we study $T^{2}$-equivariant deformations of $Z^{\prime}$.

Lemma 4.10. There exists a $T^{2}$-equivariant, commutative and exact diagram:

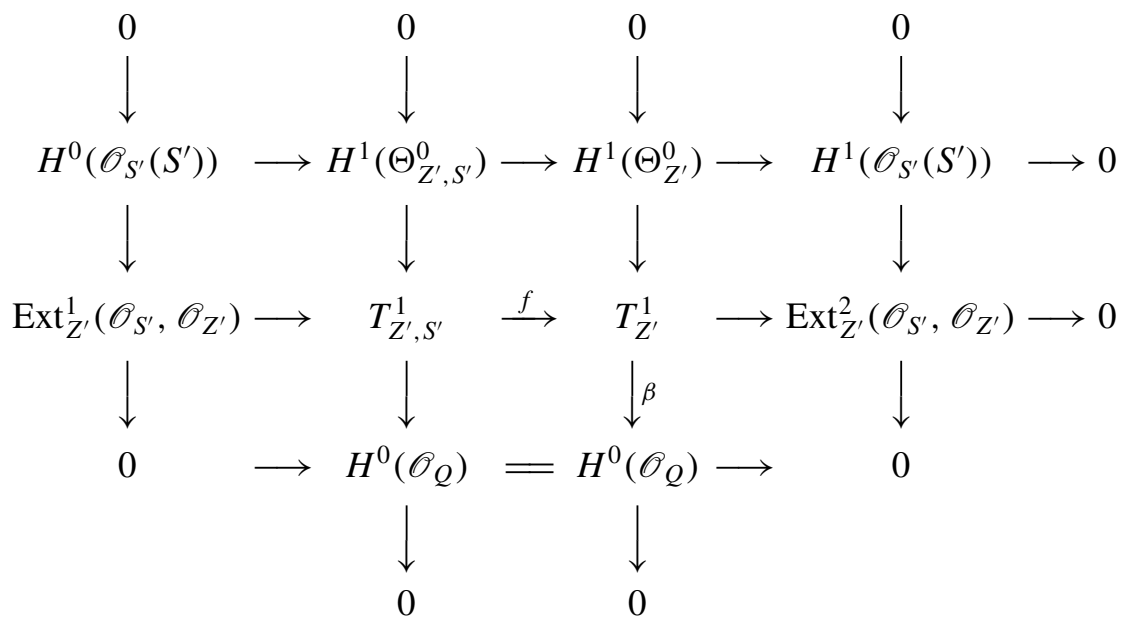

Proof. In the course of the proof of Lemma 4.8 we have proved that there exists a $T^{2}$-equivariant exact sequence (see (20))

$$
0 \longrightarrow \Theta_{Z^{\prime}, S^{\prime}}^{0} \longrightarrow \Theta_{Z^{\prime}}^{0} \longrightarrow \mathscr{O}_{S^{\prime}}\left(S^{\prime}\right) \longrightarrow 0 .
$$

The first row is the cohomology exact sequence of this with the aid of Lemma 4.9. The second row is obtained by applying the functor $\operatorname{Hom}\left(\cdot, \mathscr{O}_{Z^{\prime}}\right)$ to $(19)$ 
and using Lemma 4.9. The first and fourth columns are consequences of (18), (17), (15) and the spectral sequence

$$
E_{2}^{p, q}:=H^{p}\left(Z^{\prime}, \mathscr{E} x t_{\mathscr{O}_{Z^{\prime}}}^{q}\left(\mathscr{O}_{S^{\prime}}, \mathscr{O}_{Z^{\prime}}\right)\right) \Rightarrow \operatorname{Ext}_{Z^{\prime}}^{p+q}\left(\mathscr{O}_{S^{\prime}}, \mathscr{O}_{Z^{\prime}}\right) .
$$

The second and third columns are nothing but Lemmas 4.9 and 4.6 respectively. Finally, $T^{2}$-equivariance of the diagram follows from $T^{2}$-invariance of the divisor $S^{\prime}$, and the commutativity follow from naturality.

In view of the above diagram our next aim is to calculate $H^{1}\left(\mathscr{O}_{S^{\prime}}\left(S^{\prime}\right)\right)^{T^{2}}$.

Lemma 4.11. $H^{i}\left(Z^{\prime}, \mathscr{O}_{Z^{\prime}}\right)=0$ for any $i \geq 1$.

Proof. Simply connectedness implies $H^{1}\left(Z, \mathscr{O}_{Z}\right)=0$ for any twistor space $Z$ over $n \mathrm{CP}^{2}$ [12]. On the other hand by Serre duality we have for $j=1,2$

$$
\operatorname{dim} H^{i}\left(Z_{j}, \mathscr{O}_{Z_{j}}\right)=\operatorname{dim} H^{3-i}\left(Z_{j}, K_{Z_{j}}\right) \quad \text { for } \quad 0 \leq i \leq 3 .
$$

Now by Remark 2.2 we may use vanishing theorem of Hitchin to get $H^{1}\left(Z_{j}, K_{Z_{j}}\right)$ $=0$ for $j=1,2$. Further since $K_{Z_{j}}$ has degree (-4) on each twistor line, we have $H^{0}\left(Z_{j}, K_{Z_{j}}\right)=0$. So by (22) we obtain for $j=1,2$

$$
H^{i}\left(Z_{j}, \mathscr{O}_{Z_{j}}\right)=0 \quad \text { for } \quad i \geq 1,
$$

which imply (by using Leray spectral sequence)

$$
H^{i}\left(Z_{j}^{\prime}, \mathscr{O}_{Z_{j}^{\prime}}\right)=0 \quad \text { for } \quad i \geq 1 .
$$

Let

$$
0 \longrightarrow \mathscr{O}_{Z^{\prime}} \longrightarrow v_{*}\left(\mathscr{O}_{Z_{1}^{\prime}} \oplus \mathscr{O}_{Z_{2}^{\prime}}\right) \longrightarrow \mathscr{O}_{Q} \longrightarrow 0
$$

be the normalization sequence of $Z^{\prime}$. By Leray spectral sequence, we have

$$
H^{i}\left(Z^{\prime}, v_{*}\left(\mathscr{O}_{Z_{1}^{\prime}} \oplus \mathscr{O}_{Z_{2}^{\prime}}\right)\right) \simeq H^{i}\left(Z_{1}^{\prime}, \mathscr{O}_{Z_{1}^{\prime}}\right) \oplus H^{i}\left(Z_{2}^{\prime}, \mathscr{O}_{Z_{2}^{\prime}}\right)
$$

for any $i \geq 0$. The claim of the lemma follows from this and (23) and the cohomology exact sequence of (24).

LeMma 4.12. There exists a $T^{2}$-equivariant isomorphism

$$
H^{1}\left(S^{\prime}, \mathscr{O}_{S^{\prime}}\left(S^{\prime}\right)\right) \simeq H^{1}\left(Z^{\prime}, \mathscr{O}_{Z^{\prime}}\left(S^{\prime}\right)\right) .
$$

Proof. We have a standard exact sequence

$$
0 \longrightarrow \mathscr{O}_{Z^{\prime}} \longrightarrow \mathscr{O}_{Z^{\prime}}\left(S^{\prime}\right) \longrightarrow \mathscr{O}_{S^{\prime}}\left(S^{\prime}\right) \rightarrow 0
$$


which is $T^{2}$-equivariant. The associated cohomology sequence and Lemma 4.11 imply the claim.

LEMma 4.13. Let $Z$ be a twistor space over $n \mathrm{CP}^{2}$ with a $T^{2}$-action satisfying Condition $(A)$ and $F$ be the holomorphic line bundle satisfying $F^{\otimes 2} \simeq-K_{Z}$. Then we have $H^{1}(Z, F)^{T^{2}}=0$.

Proof. Let $S \in|F|^{T^{2}}$ be any real irreducible member. Then $S$ is nonsingular toric surface (Remark 2.9) and we have a $T^{2}$-equivariant exact sequence

$$
0 \longrightarrow \mathscr{O}_{Z} \longrightarrow F \longrightarrow-K_{S} \longrightarrow 0 .
$$

As mentioned in the proof of Lemma 4.11, we have $H^{1}\left(\mathscr{O}_{Z}\right)=H^{2}\left(\mathscr{O}_{Z}\right)=$ 0 . So we get a $T^{2}$-equivariant isomorphism $H^{1}(Z, F) \simeq H^{1}\left(S,-K_{S}\right)$. By Lemma 1.6 we have $H^{1}\left(S,-K_{S}\right)^{T^{2}}=0$. Hence we get $H^{1}(Z, F)^{T^{2}}=0$, as desired.

Proof of Proposition 4.1. It suffices to prove that the $T^{2}$-fixed part of the forgetting map $f: T_{Z^{\prime}, S^{\prime}}^{1} \rightarrow T_{Z^{\prime}}^{1}$ is surjective. By Lemmas 4.10 and 4.12 this is equivalent to $H^{1}\left(\mathscr{O}_{Z^{\prime}}\left(S^{\prime}\right)\right)^{T^{2}}=0$, which we now prove.

Recalling that $S^{\prime}=S_{1}^{\prime} \cup\left(D_{2}^{\prime} \amalg \bar{D}_{2}^{\prime}\right)$, we have a $T^{2}$-equivariant exact sequence

$$
0 \rightarrow \mathscr{O}_{Z^{\prime}}\left(S^{\prime}\right) \rightarrow v_{*}\left(\mathscr{O}_{Z_{1}^{\prime}}\left(S_{1}^{\prime}\right) \oplus \mathscr{O}_{Z_{2}^{\prime}}\left(D_{2}^{\prime}+\bar{D}_{2}^{\prime}\right)\right) \rightarrow \mathscr{O}_{Q}(l+\bar{l}) \rightarrow 0,
$$

where we set $l:=S_{1}^{\prime} \cap D_{2}^{\prime}$ and $\bar{l}:=S_{1}^{\prime} \cap \bar{D}_{2}^{\prime}$. By Leray spectral sequence, we obtain

$$
H^{i}\left(v_{*}\left(\mathscr{O}_{Z_{1}^{\prime}}\left(S_{1}^{\prime}\right) \oplus \mathscr{O}_{Z_{2}^{\prime}}\left(D_{2}^{\prime}+\bar{D}_{2}^{\prime}\right)\right)\right) \simeq H^{i}\left(\mathscr{O}_{Z_{1}^{\prime}}\left(S_{1}^{\prime}\right)\right) \oplus H^{i}\left(\mathscr{O}_{Z_{2}^{\prime}}\left(D_{2}^{\prime}+\bar{D}_{2}^{\prime}\right)\right)
$$

for any $i \geq 0$. On the other hand since $T^{2}$ acts each factor of $Q \simeq \mathrm{CP}^{1} \times \mathrm{CP}^{1}$ non-trivially, we have $H^{0}\left(\mathscr{O}_{Q}(l+\bar{l})\right)^{T^{2}} \simeq \mathrm{C}$. Hence the restriction map

$$
H^{0}\left(\mathscr{O}_{Z_{2}^{\prime}}\left(D_{2}^{\prime}+\bar{D}_{2}^{\prime}\right)\right)^{T^{2}} \longrightarrow H^{0}\left(\mathscr{O}_{Q}(l+\bar{l})\right)^{T^{2}}
$$

is surjective (because a section of $\mathscr{O}_{Z_{2}^{\prime}}\left(D_{2}^{\prime}+\bar{D}_{2}^{\prime}\right)$ which defines the divisor $D_{2}^{\prime}+\bar{D}_{2}^{\prime}$ does not contain $Q$ ). Then because the map

$$
H^{0}\left(\mathscr{O}_{Z_{1}^{\prime}}\left(S_{1}^{\prime}\right)\right) \oplus H^{0}\left(\mathscr{O}_{Z_{2}^{\prime}}\left(D_{2}^{\prime}+\bar{D}_{2}^{\prime}\right)\right) \rightarrow H^{0}\left(\mathscr{O}_{Q}(l+\bar{l})\right)
$$

is of the form $\left.(a, b) \mapsto a\right|_{Q}-\left.b\right|_{Q}$, it follows that the $T^{2}$-fixed part of the map (26) is surjective. Therefore by $(25)$ to prove $H^{1}\left(\mathscr{O}_{Z^{\prime}}\left(S^{\prime}\right)\right)^{T^{2}}=0$ it suffices to show that

$$
H^{1}\left(Z_{1}^{\prime}, \mathscr{O}_{Z_{1}^{\prime}}\left(S_{1}^{\prime}\right)\right)^{T^{2}}=H^{1}\left(Z_{2}^{\prime}, \mathscr{O}_{Z_{2}^{\prime}}\left(D_{2}^{\prime}+\bar{D}_{2}^{\prime}\right)\right)^{T^{2}}=0 .
$$


Since $S_{1}^{\prime}$ is a member of $\left|\sigma_{1}^{*} F_{1}\right|^{T^{2}}$, we have by Leray spectral sequence a $T^{2}$-equivariant isomorphism

$$
H^{1}\left(Z_{1}^{\prime}, \mathscr{O}_{Z_{1}^{\prime}}\left(S_{1}^{\prime}\right)\right) \simeq H^{1}\left(Z_{1}, F_{1}\right) .
$$

Hence by Lemma 4.13 , we get $H^{1}\left(\mathscr{O}_{Z_{1}^{\prime}}\left(S_{1}^{\prime}\right)\right)^{T^{2}}=0$. Next since $D_{2}^{\prime}+\bar{D}_{2}^{\prime}$ is the proper transform of $D_{2}+\bar{D}_{2}$, we have

$$
\mathscr{O}_{Z_{2}^{\prime}}\left(D_{2}^{\prime}+\bar{D}_{2}^{\prime}\right) \simeq \sigma_{2}^{*} F_{2} \otimes \mathscr{O}_{Z_{2}^{\prime}}\left(-2 Q_{2}\right) .
$$

Then Leray spectral sequence shows

$$
H^{1}\left(Z_{2}^{\prime}, \mathscr{O}_{Z_{2}^{\prime}}\left(D_{2}^{\prime}+\bar{D}_{2}^{\prime}\right)\right) \simeq H^{1}\left(Z_{2}, F_{2} \otimes \mathscr{I}_{L_{2}}^{2}\right)
$$

where $\mathscr{I}_{L_{2}}$ denotes the ideal sheaf of $L_{2}$ in $Z_{2}$. We note that this is also a $T^{2}$-equivariant isomorphism. Next we consider the following exact sequences

$$
0 \longrightarrow F_{2} \otimes \mathscr{I}_{L_{2}}^{2} \longrightarrow F_{2} \otimes \mathscr{I}_{L_{2}} \longrightarrow F_{2} \otimes N_{L_{2} / Z_{2}}^{*} \longrightarrow 0,
$$

and

$$
\left.0 \longrightarrow F_{2} \otimes \mathscr{I}_{L_{2}} \longrightarrow F_{2} \longrightarrow F_{2}\right|_{L_{2}} \longrightarrow 0
$$

both of which are $T^{2}$-equivariant. Now we show that $H^{0}\left(F_{2} \otimes N_{L_{2} / Z_{2}}^{*}\right)^{T^{2}}=0$. Since $D_{2}+\bar{D}_{2}$ is a member of of $\left|F_{2}\right|^{T^{2}}$ and $D_{2} \cap \bar{D}_{2}=L_{2}$ (transversally), we have $T^{2}$-equivariant isomorphisms

$$
\begin{aligned}
\left.F_{2}\right|_{L_{2}} & \left.\left.\left.\simeq\left(\mathscr{O}_{Z_{2}}\left(D_{2}+\bar{D}_{2}\right)\right)\right|_{L_{2}} \simeq\left(\mathscr{O}_{Z_{2}}\left(D_{2}\right)\right)\right|_{L_{2}} \otimes\left(\mathscr{O}_{Z_{2}}\left(\bar{D}_{2}\right)\right)\right|_{L_{2}} \\
& \left.\left.\simeq\left(\mathscr{O}_{\bar{D}_{2}}\left(D_{2}\right)\right)\right|_{L_{2}} \otimes\left(\mathscr{O}_{D_{2}}\left(\bar{D}_{2}\right)\right)\right|_{L_{2}} \simeq N_{L_{2} / D_{2}} \otimes N_{L_{2} / \bar{D}_{2}} .
\end{aligned}
$$

Therefore we get $T^{2}$-equivariant isomorphisms

$$
\begin{aligned}
F_{2} \otimes N_{L_{2} / Z_{2}}^{*} & \simeq\left(N_{L_{2} / D_{2}} \otimes N_{L_{2} / \bar{D}_{2}}\right) \otimes\left(N_{L_{2} / D_{2}}^{*} \oplus N_{L_{2} / \bar{D}_{2}}^{*}\right) \\
& \simeq N_{L_{2} / D_{2}} \oplus N_{L_{2} / \bar{D}_{2}} .
\end{aligned}
$$

The $T^{2}$-action on $H^{0}\left(N_{L_{2} / D_{2}} \oplus N_{L_{2} / \bar{D}_{2}}\right)$ can be explicitly calculated to be

$$
\left(z_{1}, z_{2}, w_{1}, w_{2}\right) \stackrel{(s, t)}{\longmapsto}\left(s t z_{1}, s^{-1} t z_{2}, s^{-1} t^{-1} w_{1}, s t^{-1} w_{2}\right)
$$

for a coordinate $\left(z_{1}, z_{2}, w_{1}, w_{2}\right)$ on $H^{0}\left(N_{L_{2} / D_{2}}\right) \oplus H^{0}\left(N_{L_{2} / \bar{D}_{2}}\right) \simeq C^{2} \oplus C^{2}$ and $(s, t) \in T^{2}=U(1) \times U(1)$. In particular, we have $H^{0}\left(N_{L_{2} / D_{2}} \oplus N_{L_{2} / \bar{D}_{2}}\right)^{T^{2}}=$ 0 . Therefore we get $H^{0}\left(F_{2} \otimes N_{L_{2} / Z_{2}}^{*}\right)^{T^{2}}=0$. On the other hand, since 
$F_{2} \otimes N_{L_{2} / Z_{2}}^{*} \simeq \mathscr{O}_{L_{2}}(1)^{\oplus 2}$, we have $H^{1}\left(F_{2} \otimes N_{L_{2} / Z_{2}}^{*}\right)=0$. Therefore, by the cohomology exact sequence of (28), we obtain

$$
H^{1}\left(F_{2} \otimes \mathscr{I}_{L_{2}}^{2}\right)^{T^{2}} \simeq H^{1}\left(F_{2} \otimes \mathscr{I}_{L_{2}}\right)^{T^{2}} .
$$

Next we show that the restriction map $H^{0}\left(F_{2}\right)^{T^{2}} \rightarrow H^{0}\left(\left.F_{2}\right|_{L_{2}}\right)^{T^{2}}$ is surjective. Using (30), we can explicitly calculate the $T^{2}$-action on $H^{0}\left(\left.F_{2}\right|_{L_{2}}\right) \simeq \mathrm{C}^{3}$ to be

$$
\left(u_{1}, u_{2}, u_{3}\right) \stackrel{(s, t)}{\longmapsto}\left(s^{2} u_{1}, u_{2}, s^{-2} u_{3}\right)
$$

for a coordinate $\left(u_{1}, u_{2}, u_{3}\right)$ on $C^{3}$ and $(s, t) \in T^{2}=U(1) \times U(1)$. Thus we have $H^{0}\left(\left.F_{2}\right|_{L_{2}}\right)^{T^{2}} \simeq \mathrm{C}$ and is generated by a section whose zero locus is the fixed point set of the $T^{2}$-action on $L_{2}$. On the other hand since any member of the pencil $\left|F_{2}\right|^{T^{2}}$ other than $D_{2}^{\prime}+\bar{D}_{2}^{\prime}$ does not contain $L_{2}$ by Proposition 2.8 (v), the image of the map $H^{0}\left(F_{2}\right)^{T^{2}} \rightarrow H^{0}\left(\left.F_{2}\right|_{L_{2}}\right)^{T^{2}}$ is not zero. This implies the surjectivity. Therefore the cohomology exact sequence of (29) together with Lemma 4.13 shows that $H^{1}\left(F_{2} \otimes \mathscr{I}_{L_{2}}\right)^{T^{2}} \simeq H^{1}\left(F_{2}\right)^{T^{2}}=0$. Hence by (27) and (32), we get $H^{1}\left(\Theta_{Z_{2}^{\prime}}\left(D_{2}^{\prime}+\bar{D}_{2}^{\prime}\right)\right)^{T^{2}}=0$. Consequently we have $H^{1}\left(Z^{\prime}, \mathscr{O}_{Z^{\prime}}\left(S^{\prime}\right)\right)^{T^{2}}=0$ and the claim of the proposition follows.

Our next task is to study relation of deformations of the pair $\left(Z^{\prime}, S^{\prime}\right)$ and those of $S^{\prime}$. The following $T^{2}$-equivariant exact sequence is the key:

$$
0 \longrightarrow \Omega_{Z^{\prime}, S^{\prime}} \longrightarrow \Omega_{Z^{\prime}}\left(S^{\prime}\right) \longrightarrow \Omega_{S^{\prime}}\left(S^{\prime}\right) \longrightarrow 0 .
$$

Here we set $\Omega_{Z^{\prime}}\left(S^{\prime}\right):=\Omega_{Z^{\prime}} \otimes_{\mathscr{O}_{Z^{\prime}}} \mathscr{O}_{Z^{\prime}}\left(S^{\prime}\right)$ and $\Omega_{S^{\prime}}\left(S^{\prime}\right):=\Omega_{S^{\prime}} \otimes_{\mathscr{O}_{S^{\prime}}} \mathscr{O}_{S^{\prime}}\left(S^{\prime}\right)$.

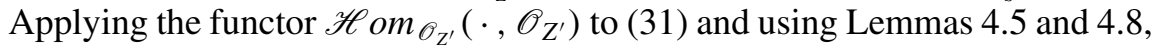
we get the long exact sequence

$$
\begin{aligned}
& 0 \longrightarrow \Theta_{Z^{\prime}}^{0}\left(-S^{\prime}\right) \longrightarrow \Theta_{Z^{\prime}, S^{\prime}}^{0} \longrightarrow \mathscr{E} x t_{\mathscr{O}_{Z^{\prime}}}^{1}\left(\Omega_{S^{\prime}}\left(S^{\prime}\right), \mathscr{O}_{Z^{\prime}}\right) \\
\longrightarrow & \Theta_{Z^{\prime}}^{1}\left(-S^{\prime}\right) \longrightarrow \Theta_{Z^{\prime}, S^{\prime}}^{1} \longrightarrow \mathscr{E} x t_{\mathscr{O}_{Z^{\prime}}}^{2}\left(\Omega_{S^{\prime}}\left(S^{\prime}\right), \mathscr{O}_{Z^{\prime}}\right) \longrightarrow \Theta_{Z^{\prime}}^{2}\left(-S^{\prime}\right)=0
\end{aligned}
$$

and

$$
\mathscr{E} x t_{\mathscr{O}_{Z^{\prime}}}^{i}\left(\Omega_{S^{\prime}}\left(S^{\prime}\right), \mathscr{O}_{Z^{\prime}}\right)=0
$$

for any $i \geq 3$. Here we have used the invertibility of $\mathscr{O}_{Z^{\prime}}\left(S^{\prime}\right)$ to obtain

$$
\mathscr{E} x t_{\mathscr{O}_{Z^{\prime}}}^{q}\left(\Omega_{Z^{\prime}}\left(S^{\prime}\right), \mathscr{O}_{Z^{\prime}}\right) \simeq \Theta_{Z^{\prime}}^{q}\left(-S^{\prime}\right) .
$$

On the other hand, we have $\Theta_{Z^{\prime}, S^{\prime}}^{1} \simeq \Theta_{Z^{\prime}}^{1}$ by Lemma 4.8. Therefore the map $\Theta_{Z^{\prime}}^{1}\left(-S^{\prime}\right) \rightarrow \Theta_{Z^{\prime}, S^{\prime}}^{1}$ in (33) is injective and (33) reduces to the following two short exact sequences:

$$
0 \longrightarrow \Theta_{Z^{\prime}}^{0}\left(-S^{\prime}\right) \longrightarrow \Theta_{Z^{\prime}, S^{\prime}}^{0} \longrightarrow \mathscr{E} x t_{\mathscr{Z}_{Z^{\prime}}}^{1}\left(\Omega_{S^{\prime}}\left(S^{\prime}\right), \mathscr{O}_{Z^{\prime}}\right) \longrightarrow 0,
$$




$$
0 \longrightarrow \Theta_{Z^{\prime}}^{1}\left(-S^{\prime}\right) \longrightarrow \Theta_{Z^{\prime}, S^{\prime}}^{1} \longrightarrow \mathscr{E} x t_{\mathscr{O}_{Z^{\prime}}}^{2}\left(\Omega_{S^{\prime}}\left(S^{\prime}\right), \mathscr{O}_{Z^{\prime}}\right) \longrightarrow 0
$$

By naturality, these imply there exist $T^{2}$-equivariant isomorphisms

$$
\begin{gathered}
\mathscr{E} x t_{\mathscr{O}_{Z^{\prime}}}^{1}\left(\Omega_{S^{\prime}}\left(S^{\prime}\right), \mathscr{O}_{Z^{\prime}}\right) \simeq \Theta_{S^{\prime}}^{0}, \\
\mathscr{E} x t_{\mathscr{O}_{Z^{\prime}}}^{2}\left(\Omega_{S^{\prime}}\left(S^{\prime}\right), \mathscr{O}_{Z^{\prime}}\right) \simeq \Theta_{Z^{\prime}}^{1} \mid S_{S^{\prime}}\left(\simeq \mathscr{O}_{l} \oplus \mathscr{O}_{\bar{l}}\right) .
\end{gathered}
$$

Lemma 4.14. There exists a $T^{2}$-equivariant, commutative and exact diagram:

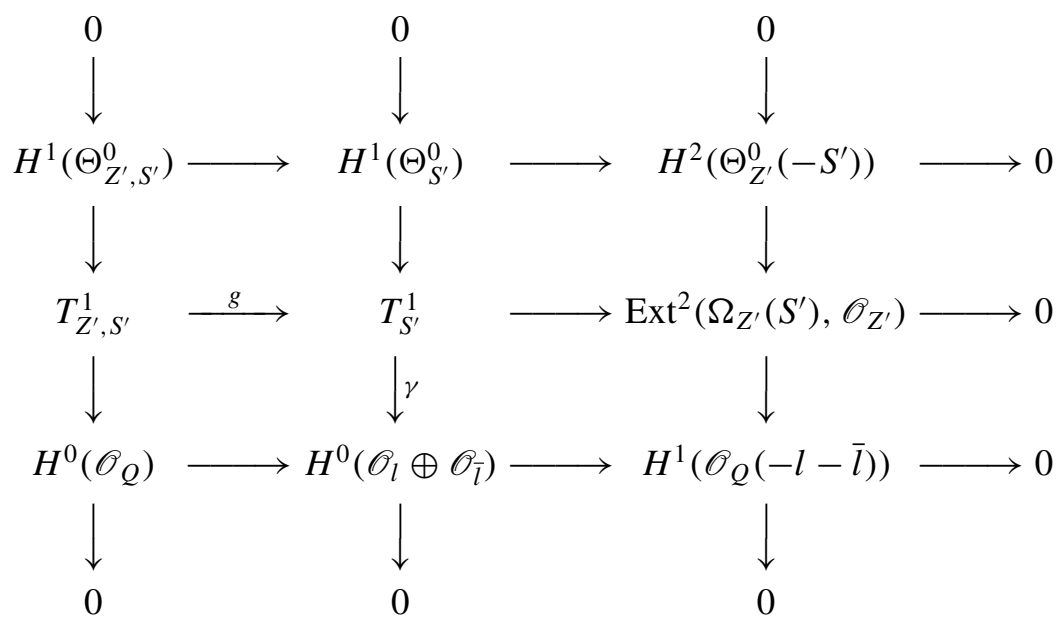

Proof. The first row is induced by (21) (or (35)), with the aid of Lemma 4.9. The third row is the cohomology sequence of (36). The third column is obtained by local to global spectral sequence for $\operatorname{Ext}_{Z^{\prime}}^{i}\left(\Omega_{Z^{\prime}}\left(S^{\prime}\right), \mathscr{O}_{Z^{\prime}}\right)$. The first and the second columns were already proved in Lemmas 4.9 and 4.2 respectively.

By (34), (37) and (38) local to global spectral sequence associated to the hypercohomology groups $\operatorname{Ext}_{Z^{\prime}}^{i}\left(\Omega_{S^{\prime}}\left(S^{\prime}\right), \mathscr{O}_{Z^{\prime}}\right)$ induces a $T^{2}$-equivariant exact sequence

$$
\begin{aligned}
0 \longrightarrow H^{1}\left(\Theta_{S^{\prime}}^{0}\right) \longrightarrow \operatorname{Ext}_{Z^{\prime}}^{2}\left(\Omega_{S^{\prime}}\left(S^{\prime}\right), \mathscr{O}_{Z^{\prime}}\right) & \\
& \longrightarrow H^{0}\left(\mathscr{O}_{l} \oplus \mathscr{O}_{\bar{l}}\right) \longrightarrow H^{2}\left(\Theta_{S^{\prime}}^{0}\right)=0 .
\end{aligned}
$$

Here, we have used Lemma 4.2. The $T^{2}$-action determines a splitting of this sequence. On the other hand, the exact sequence in Lemma 4.2 also has a splitting arising from the $T^{2}$-action. That is, $T_{S^{\prime}}^{1} \simeq H^{1}\left(\Theta_{S^{\prime}}^{0}\right) \oplus H^{0}\left(\Theta_{S^{\prime}}^{1}\right)$. These induce $T^{2}$-equivariant isomorphism $\operatorname{Ext}_{Z^{\prime}}^{2}\left(\Omega_{S^{\prime}}\left(S^{\prime}\right), \mathscr{O}_{Z^{\prime}}\right) \simeq T_{S^{\prime}}^{1}$. Then the second row is obtained by applying the functor $\operatorname{Hom}_{Z^{\prime}}\left(\cdot, \mathscr{O}_{Z^{\prime}}\right)$ to (32) and 
using Lemma 4.9. Finally, $T^{2}$-equivariance of the diagram follows from $T^{2}$ invariance of the divisor $S^{\prime}$, and the commutativity follow from the naturality.

Remark 4.15. As proved in Proposition 3.3 we have $H^{2}\left(\Theta_{Z^{\prime}}^{0}\left(-S^{\prime}\right)\right)=0$. Hence the third column of the above diagram implies

$$
\operatorname{Ext}_{Z^{\prime}}^{2}\left(\Omega_{Z^{\prime}}\left(S^{\prime}\right), \mathscr{O}_{Z^{\prime}}\right) \simeq H^{1}\left(\mathscr{O}_{Q}(-l-\bar{l})\right) \simeq \mathrm{C} .
$$

In particular, $g: T_{Z^{\prime}, S^{\prime}}^{1} \rightarrow T_{S^{\prime}}^{1}$ is not surjective. (See also [7, Remark 2.D].)

Proposition 4.16 (= Proposition 3.2 (ii), (iii)). Let $Z^{\prime}, S^{\prime}$ be as in Proposition 4.1. Then $Z^{\prime}$ can be $T^{2}$-equivariantly deformed into a non-singular threefold. In such a case, $S^{\prime}$ is also deformed into a non-singular toric surface which is biholomorphic to $S_{1}^{\prime}$.

Proof. Let $\eta \in H^{0}\left(\Theta_{Z^{\prime}}^{1}\right) \simeq \mathrm{C}$ be any non-zero element. Then by Lemmas 4.6 and 4.7 there exists $\xi \in\left(T_{Z^{\prime}}^{1}\right)^{T^{2}}$ such that $\beta(\xi)=\eta$. Moreover because we have shown in the proof of Proposition 4.1 the surjectivity of $f: T_{Z^{\prime}, S^{\prime}}^{1} \rightarrow T_{Z^{\prime}}^{1}$ (in the diagram of Lemma 4.10), there exists $\hat{\xi} \in\left(T_{Z^{\prime}, S^{\prime}}^{1}\right)^{T^{2}}$ such that $f(\hat{\xi})=\xi$. Let $\Delta \subseteq C$ be a small disk around zero and $\{p: \mathscr{Z} \rightarrow \Delta, q: \mathscr{S} \rightarrow$ $\Delta, \mathscr{S} \hookrightarrow \mathscr{Z}\}$ be a $T^{2}$-equivariant deformation of the pair $\left(Z^{\prime}, S^{\prime}\right)$ whose Kodaira-Spencer class is $\hat{\xi}$. Then the assumption on $\eta$ and a commutativity of the diagram in Lemma 4.10 imply $Z_{t}:=p^{-1}(t)$ is non-singular for $t \neq 0$. Furthermore, a commutativity of the diagram in Lemma 4.14 implies that both of the two factors of $\gamma(g(\hat{\xi})) \in H^{0}\left(\mathscr{O}_{l} \oplus \mathscr{O}_{\bar{l}}\right)$ are non-zero. This implies that $g(\hat{\xi}) \in\left(T_{S^{\prime}}^{1}\right)^{T^{2}}$ is not tangent to $B_{1}$ nor $B_{2}$ in Proposition 4.4 and hence the fiber $S_{t}:=q^{-1}(t)$ is non-singular toric surface biholomorphic to $S_{1}^{\prime}$. Hence all of the claims of the proposition are verified.

\section{Relation between the orbit data}

In this section we determine relation between the weighted dual graphs of $T^{2}$-actions on $n \mathrm{CP}^{2}$ (cf. §1.1) and those of toric surfaces (cf. §1.2) in $|F|^{T^{2}}$ of Pedersen-Poon twistor spaces. This in particular implies (iv) of Main Theorem in Introduction.

Let $\alpha: T^{2} \times n \mathrm{CP}^{2} \rightarrow n \mathrm{CP}^{2}$ be any effective $T^{2}$-action and $\left(a_{1}, a_{2}, \ldots, a_{k}\right)$ with $k:=n+2$ be the dual graph of $\alpha$. Let $A_{i}(1 \leq i \leq k)$ be the set of $T^{2}$ invariant spheres which satisfy $A_{i}^{2}=a_{i}$ (hence $\left\{A_{i}\right\}$ is arranged in the cyclic order), and $p_{i}=A_{i-1} \cap A_{i}, 1 \leq i \leq k$, the $T^{2}$-fixed points, where subscripts are counted modulo $k$. Let $Z$ be a Pedersen-Poon twistor space associated to $\alpha$. Let $\left\{D_{i}+\bar{D}_{i} \mid 1 \leq i \leq k\right\}$ be the set of reducible members of $|F|^{T^{2}}$, where $D_{i}$ (and $\bar{D}_{i}$ ) is a non-singular toric surface (see (i)-(iii) of Main Theorem). We assume that $\left\{D_{i}\right\}$ is arranged as in Proposition 2.8 (iv); that is, $D_{i} \cap \bar{D}_{i}=L_{i}$ for 
any $1 \leq i \leq k$, where $L_{i}$ is the twistor line over $p_{i}$. Let $S \in|F|^{T^{2}}$ be a generic member, which is also a non-singular toric surface. Let $C=\sum_{i=1}^{k}\left(C_{i}+\bar{C}_{i}\right)$ be the base locus of the pencil $|F|^{T^{2}}$, where we assume $C_{i}$ are arranged so as to satisfy $C_{i-1} \cap C_{i} \subseteq L_{i}$ for any $1 \leq i \leq k$.

Main result of this section is the following

Proposition 5.1 (See also [5]). Let $\left(b_{1}, b_{2}, \ldots, b_{2 k}\right)$ and $\left(c_{1}, c_{2}, \ldots, c_{k+1}\right)$ be the dual graphs of $S$ and $D_{i}(1 \leq i \leq k)$ respectively. Then we have

$$
b_{j}=\left\{\begin{array}{ll}
-a_{j}, & 1 \leq j \leq k, \\
-a_{j-k}, & k+1 \leq j \leq 2 k
\end{array} \quad \text { and } \quad c_{j}= \begin{cases}1, & j=i, \\
-a_{j}+1, & j=i \pm 1, \\
-a_{j}, & \text { otherwise }\end{cases}\right.
$$

Proof. First we show the statement for $S$. In this proof we use the following conventions: subscripts attached to $r, s, m^{\prime \prime}$ and $n^{\prime \prime}$ are counted modulo $2 k$ and subscripts attached to $m, n$ and $a$ are counted modulo $k$. Let $\Delta$ be a fan to which $S$ corresponds and $\left\{\tau_{i}=\mathrm{R}_{\geq 0}\left(r_{i}, s_{i}\right) \mid 1 \leq i \leq 2 k\right\}$ be the set of onedimensional cones of $\Delta$. $\left\{\left(r_{j}, s_{j}\right)\right\}$ satisfy $r_{i} s_{j+1}-r_{j+1} s_{i}=1$ for $1 \leq j \leq 2 k$. Then what we have to prove is, by (2) (in §1.2), that there exists an integer $l$ such that

$$
-r_{j-1} s_{j+1}+r_{j+1} s_{j-1}=-a_{-j+l}
$$

holds for every $1 \leq j \leq 2 k$. (Recall that the direction of points on the graphs of $\alpha$ and $S$ are reverse to each other.)

It is obvious that $\left.\pi\right|_{C}$ induces an unramified double covering map

$$
C=\sum_{j=1}^{k}\left(C_{j}+\bar{C}_{j}\right) \longrightarrow A:=\cup_{j=1}^{k} A_{j}
$$

with $\pi\left(C_{j}\right)=\pi\left(\bar{C}_{j}\right)=A_{j}$. Of course this is a $T^{2}$-equivariant map and therefore the stabilizer of $C_{j}$ and $\bar{C}_{j}$ are the same as that of $A_{j}$. Let $\left\{\left(m_{j}, n_{j}\right) \mid\right.$ $1 \leq j \leq k\}$ be the stabilizer data which is obtained from $\left(a_{j}\right)$ by using the procedure explained in $\S 2.1\left(\left(a_{j}\right) \longmapsto\left\{\left(m_{j}, n_{j}\right)\right\}\right)$. They satisfy

$$
\begin{aligned}
a_{j} & =-\left|\begin{array}{ll}
m_{j-1} & m_{j} \\
n_{j-1} & n_{j}
\end{array}\right|\left|\begin{array}{cc}
m_{j} & m_{j+1} \\
n_{j} & n_{j+1}
\end{array}\right|\left|\begin{array}{cc}
m_{j-1} & m_{j+1} \\
n_{j-1} & n_{j+1}
\end{array}\right| \\
& = \begin{cases}-m_{j-1} n_{j+1}+m_{j+1} n_{j-1}, & 2 \leq j \leq k-1, \\
m_{k} n_{2}-m_{2} n_{k}, & j=1, \\
m_{k-1} n_{1}-m_{1} n_{k-1}, & j=k .\end{cases}
\end{aligned}
$$


In order for these stabilizer data to fit in the setting of toric surface geometry explained in $\S 2.2$, first we set

$$
\left(\begin{array}{c}
m_{j}^{\prime} \\
n_{j}^{\prime}
\end{array}\right):=\left(\begin{array}{c}
m_{j} \\
n_{j}
\end{array}\right) \quad \text { and } \quad\left(\begin{array}{c}
m_{k+j}^{\prime} \\
n_{k+j}^{\prime}
\end{array}\right):=\left(\begin{array}{c}
-m_{j} \\
-n_{j}
\end{array}\right)
$$

for $1 \leq j \leq k$. Further (to reverse the direction of the rotation of numbering) we set

$$
\left(\begin{array}{c}
m_{j}^{\prime \prime} \\
n_{j}^{\prime \prime}
\end{array}\right):=\left(\begin{array}{c}
m_{k-j+1}^{\prime} \\
n_{k-j+1}^{\prime}
\end{array}\right) \quad \text { and } \quad\left(\begin{array}{c}
m_{k+j}^{\prime \prime} \\
n_{k+j}^{\prime \prime}
\end{array}\right):=\left(\begin{array}{c}
m_{2 k-j+1}^{\prime} \\
n_{2 k-j+1}^{\prime}
\end{array}\right)
$$

for $1 \leq j \leq k$. Then it is readily checked that these new data satisfy

$$
\left|\begin{array}{cc}
m_{j}^{\prime \prime} & m_{j+1}^{\prime \prime} \\
n_{j}^{\prime \prime} & n_{j+1}^{\prime \prime}
\end{array}\right|=1
$$

for $1 \leq j \leq 2 k$, where we have $m_{2 k+1}^{\prime \prime}=m_{1}^{\prime \prime}$ and $n_{2 k+1}^{\prime \prime}=n_{1}^{\prime \prime}$ by the conventions. Then by Lemma 1.5 we have

$$
\left(\begin{array}{c}
r_{j} \\
s_{j}
\end{array}\right)=\left(\begin{array}{c}
-n_{j}^{\prime \prime} \\
m_{j}^{\prime \prime}
\end{array}\right)
$$

for any $1 \leq j \leq 2 k$. Hence we have

$$
\begin{aligned}
-r_{j-1} s_{j+1}+r_{j+1} s_{j-1} & \\
& =n_{j-1}^{\prime \prime} m_{j+1}^{\prime \prime}-n_{j+1}^{\prime \prime} m_{j-1}^{\prime \prime} \\
& = \begin{cases}n_{k-j+2}^{\prime} m_{k-j}^{\prime}-n_{k-j}^{\prime} m_{k-j+2}^{\prime}, & 2 \leq j \leq k-1, \\
n_{3 k-j+2}^{\prime} m_{3 k-j}^{\prime}-n_{3 k-j}^{\prime} m_{3 k-j+2}^{\prime}, & k+2 \leq j \leq 2 k-1,\end{cases} \\
& = \begin{cases}n_{k-j+2} m_{k-j}-n_{k-j} m_{k-j+2}, & 2 \leq j \leq k-1, \\
n_{2 k-j+2} m_{2 k-j}-n_{2 k-j} m_{2 k-j+2}, & k+2 \leq j \leq 2 k-1,\end{cases} \\
& = \begin{cases}-a_{k-j+1}, & 2 \leq j \leq k-1, \\
-a_{2 k-j+1}, & k+2 \leq j \leq 2 k-1 .\end{cases}
\end{aligned}
$$

On the other hand, if we take $j=1$,

$$
\begin{aligned}
-r_{2 k} s_{2}+r_{2} s_{2 k} & =n_{2 k}^{\prime \prime} m_{2}^{\prime \prime}-n_{2}^{\prime \prime} m_{2 k}^{\prime \prime}=n_{k+1}^{\prime} m_{k-1}^{\prime}-n_{k-1}^{\prime} m_{k+1}^{\prime} \\
& =-n_{1} m_{k-1}+n_{k-1} m_{1}=-a_{k} .
\end{aligned}
$$


If we take $j=k$ we have

$$
\begin{aligned}
-r_{k-1} s_{k+1}+r_{k+1} s_{k-1} & =n_{k-1}^{\prime \prime} m_{k+1}^{\prime \prime}-n_{k+1}^{\prime \prime} m_{k-1}^{\prime \prime}=n_{2}^{\prime} m_{2 k}^{\prime}-n_{2 k}^{\prime} m_{2}^{\prime} \\
& =-n_{2} m_{k}+n_{k} m_{2}=-a_{1} .
\end{aligned}
$$

In a similar way, we have

$$
-r_{k} s_{k+2}+r_{k+2} s_{k}=-a_{k}, \quad(j=k+1),
$$

and

$$
-r_{2 k-1} s_{1}+r_{1} s_{2 k-1}=-a_{1}, \quad(j=2 k) .
$$

Therefore if we set $l:=k+1(\equiv 1)$, then (39) holds. Thus the claim for $S$ is proved.

The claim for $D_{i}$ is easier if we notice that $D_{i}$ is obtained as a $T^{2}$-equivariant connected sum of $\left(n \mathrm{CP}^{2}, \alpha\right)$ and $\left(\overline{\mathrm{CP}}^{2}, \alpha_{1}\right)$ at $p_{i}$ (here $\overline{\mathrm{CP}}^{2}$ denotes the complex projective plane with the complex orientation reversed): We can use the same argument used in the proof of Proposition 1.3. So we omit it.

\section{REFERENCES}

1. Atiyah, M., Hitchin, N., Singer, I., Self-duality in four-dimensional Riemannian geometry, Proc. Roy. Soc. London, Ser. A 362 (1978), 425-461.

2. Cathelineau, J. L., Déformation Équivariantes d'espaces analytiques complexes compacts, Ann. Scient. Éc. Norm. Sup. 4e serie 11 (1978), 391-406.

3. Donaldson, S. K., Friedman, R., Connected sums of self-dual manifolds and deformations of singular spaces, Nonlinearity 2 (1989), 197-239.

4. Friedman, R., Global smoothings of varieties with normal crossings, Ann. of Math. 118 (1983), 75-114.

5. Fujiki, A., Compact self-dual manifolds with torus actions, J. Differential Geom. 55 (2000), 229-324.

6. Hitchin, N., Linear field equations on self-dual spaces, Proc. London Math. Soc. 370 (1980), 173-191.

7. Honda, N., Donaldson-Friedman construction and deformations of a triple of compact complex spaces, Osaka J. Math. 36 (1999), 641-672.

8. Honda, N. and Itoh, M., A Kummer type construction of self-dual metrics on the connected sum of four complex projective planes, J. Math. Soc. Japan 52 (2000), 139-160.

9. Horikawa, E., Deformations of holomorphic maps. III, Math. Ann. 222 (1976), 275-282.

10. Joyce, D., Explicit construction of self-dual 4-manifolds, Duke Math. J. 77 (1995), 519-552.

11. LeBrun, C., Explicit self-dual metrics on $\mathrm{CP}_{2} \# \ldots \# \mathrm{CP}_{2}$, J. Differential Geom. 34 (1991), 223-253.

12. LeBrun, C., Twistors, Kähler manifolds and bimeromorphic geometry. I, J. Amer. Math. Soc. 5 (1992), 289-316.

13. Oda, T., Convex Bodies and Algebraic Geometry, Ergeb. Math. Grenzgeb. (3) 15.

14. Orlik, P., Raymond, F., Actions of the torus on 4-manifolds I, Trans. Amer. Math. Soc. 152 (1970), 531-559. 
15. Pedersen, H., Poon, Y. S., Self-duality and differentiable structures on the connected sum of complex projective planes, Proc. Amer. Math. Soc. 121 (1994), 859-864.

16. Pedersen, H., Poon, Y. S., Equivariant connected sums of compact self-dual manifolds, Math. Ann. 301 (1995), 717-749.

17. Pedersen, H., Poon, Y. S., Connected sums of self-dual manifolds and equivariant relative smoothings, Pacific J. Math. 191 (1999), 145-171.

18. Pontecorvo, M., Algebraic dimension of twistor spaces and scalar curvature of anti-self-dual metrics, Math. Ann. 291 (1991), 113-122.

19. Poon, Y. S., Compact self-dual manifolds of positive scalar curvature, J. Differential Geom. 24 (1986), 97-132.

20. Poon, Y. S., On the algebraic structure of twistor spaces, J. Differential Geom. 36 (1992), 451-491.

DEPARTMENT OF MATHEMATICS

GRADUATE SCHOOL OF SCIENCE

HIROSHIMA UNIVERSITY

739-8526, JAPAN

E-mail: honda@math.sci.hiroshima-u.ac.jp 